\title{
A!
}

This is an electronic reprint of the original article.

This reprint may differ from the original in pagination and typographic detail.

Paananen, T.; Koponen, T. K.; Törmä, P.; Martikainen, J. P.

\section{Noise correlations of the ultracold Fermi gas in an optical lattice}

Published in:

Physical Review A

DOI:

10.1103/PhysRevA.77.053602

Published: 08/05/2008

Document Version

Publisher's PDF, also known as Version of record

Please cite the original version:

Paananen, T., Koponen, T. K., Törmä, P., \& Martikainen, J. P. (2008). Noise correlations of the ultracold Fermi gas in an optical lattice. Physical Review A, 77(5), 1-15. [053602]. https://doi.org/10.1103/PhysRevA.77.053602

This material is protected by copyright and other intellectual property rights, and duplication or sale of all or part of any of the repository collections is not permitted, except that material may be duplicated by you for your research use or educational purposes in electronic or print form. You must obtain permission for any other use. Electronic or print copies may not be offered, whether for sale or otherwise to anyone who is not an authorised user. 


\title{
Noise correlations of the ultracold Fermi gas in an optical lattice
}

\author{
T. Paananen, ${ }^{1, *}$ T. K. Koponen, ${ }^{2,3}$ P. Törmä, ${ }^{2,3}$ and J.-P. Martikainen ${ }^{1,4}$ \\ ${ }^{1}$ Department of Physics, University of Helsinki, P.O. Box 64, 00014 University of Helsinki, Finland \\ ${ }^{2}$ Nanoscience Center, Department of Physics, P.O. Box 35, 40014 University of Jyväskylä, Finland \\ ${ }^{3}$ Department of Engineering Physics, Helsinki University of Technology, P.O. Box 5100, 02015 HUT, Finland \\ ${ }^{4}$ Nordita, Roslagstullsbacken 23, 10691 Stockholm, Sweden
}

(Received 7 January 2008; revised manuscript received 17 March 2008; published 7 May 2008)

\begin{abstract}
In this paper we study the density noise correlations of the two component Fermi gas in optical lattices. Three different types of phases, the BCS state (Bardeen, Cooper, and Schieffer), the FFLO state (Fulde, Ferrel, Larkin, and Ovchinnikov), and the BP (breach pair) state are considered. We show how these states differ in their noise correlations. The noise correlations are calculated not only at zero temperature, but also at nonzero temperatures paying particular attention to how much the finite temperature effects might complicate the detection of different phases. Since one-dimensional systems have been shown to be very promising candidates to observe FFLO states, we apply our results also to the computation of correlation signals in a onedimensional lattice. We find that the density noise correlations reveal important information about the structure of the underlying order parameter as well as about the quasiparticle dispersions.
\end{abstract}

DOI: 10.1103/PhysRevA.77.053602

PACS number(s): 03.75.Ss

\section{INTRODUCTION}

Recent studies in the experiments on ultracold Fermi gases have shown great potential for elucidating longstanding problems in many different fields of physics related to strongly correlated fermions. For instance, in recent experiments [1-6] spin-density imbalanced, or polarized, Fermi gases were considered. Among other things, such systems make it possible to study pairing with mismatched Fermi surfaces, potentially leading to non-BCS- (Bardeen, Cooper, and Schieffer) type pairing such as that appearing in FFLO (Fulde, Ferrel, Larkin, and Ovchinnikov) states $[7,8]$ or BP (breach pair) states $[9,10]$ (Sarma states). These possibilities have been considered extensively in condensed-matter, nuclear, and high-energy physics [11].

In optical lattices it is possible to study many different physical problems with close analogs in the field of solid state physics. However, in contrast to many solid state systems, ultracold gases in optical lattices provide a very clean environment. In other words, these systems have very few imperfections and if imperfections are of interest, they can be introduced in a more controlled manner. In addition, since optical lattices are made with lasers, the lattice geometry is easy to vary [12-15] by changing the properties of the intersecting laser beams. For these reasons optical lattices enable one to investigate various quantum many-body physics problems, such as those related to Mott insulators, phase coherence, and superfluidity. Indeed, the possibility of a superfluid alkali-metal-atom Fermi gas in an optical lattice has been recently studied both theoretically [16-22], as well as experimentally [23]. Furthermore, Feshbach resonances, molecules of fermionic atoms, and $p$-wave interactions in a lower dimensional fermionic system have been studied using optical lattices [24-26].

Density-density correlations tell us how strongly the atomic densities at different positions are correlated. Often it

\footnotetext{
*Tomi.Paananen@helsinki.fi
}

is useful to focus on the deviation of the atomic density from its mean value and subtract the background away. In such cases one studies noise correlations, i.e., how the density fluctuations at different positions are correlated. Measuring noise correlations is a promising way to observe different phases in an optical lattice since while densities can be very similar for different phases, the noise correlations can still be very different. As an example, one can mention the famous phase transition between the superfluid Bose gas and the Mott insulator [27,28]. In the Mott insulator phase one can see the Bragg peaks in the noise correlations, but the noise correlations vanish for a Bose-Einstein condensate. Experimentally, noise correlations have been used as an indicator for different phases in optical lattices for bosons [28] and to observe fermionic antibunching for ideal fermions [29]. Also, noise correlations have been used to detect pairing correlations in an interacting Fermi gas in a harmonic trap [30].

In this paper we study noise correlations in a cloud of ultracold two component Fermi gas at finite temperatures. We are motivated by the fact that via noise correlations one can see subtle correlation effects which are not visible in the lower order correlations functions. Not only are the noise correlation signals very different for a superfluid and a normal Fermi gas, but noise correlations also differ between different types of paired states. While we compute densitydensity correlations also at zero temperature, special attention is paid to finite temperature calculations, since finite temperature effects have not been extensively discussed before. We find that although finite temperature effects do smooth out some sharp features present in zero temperature calculations, qualitative and clear quantitative differences between phases can still exist at higher temperatures. Also, we show how regions of gapless excitations are reflected in the density-density correlations. For instance, gapless excitations show up as the absence of correlations for certain momenta. Moreover, they are also reflected in the value of the maximum correlation peak height; this quantitative signature persists at finite temperature as well. 
Reducing the dimensionality of the system from three dimensions into one has been shown to favor the FFLO-type modulated order parameters in free space [31-33] as well as in a lattice [34]. This effect of dimensionality, together with the general tendency of a lattice geometry to favor the FFLO state due to nesting of the Fermi surfaces [22], makes onedimensional optical lattices a promising system to study the FFLO state. For this reason, we apply our general results on density-density correlations also to the one-dimensional (1D) system. Very recently a similar one-dimensional problem was also discussed at zero temperature using the density matrix renormalization group (DMRG) algorithm [35]. Here we compute the density-density correlations of the onedimensional system at representative points of the finite temperature phase diagram of a polarized system. The noise correlations in a one-dimensional system turn out to show differences between different states which are straightforward to interpret and contain useful information on the underlying pair-wave function as well as on the structure of the quasiparticle dispersions.

This paper is organized as follows. In Sec. II we discuss the physical system and present the Hamiltonian of the system. In this section different paired phases are also discussed In Sec. III we proceed to compute the noise correlation functions for different paired phases at zero temperature and in Sec. IV computations are generalized to nonzero temperatures. In Sec. V noise correlations in a one-dimensional lattice are discussed while making a clear connection with the computed 1D phase diagram. We end with some concluding remarks in Sec. VI.

\section{HAMILTONIAN OF THE SYSTEM}

We assume in our calculation a three-dimensional cubic optical lattice. The system is composed of a two component Fermi gas where components are different hyperfine states (for concreteness we assume ${ }^{6} \mathrm{Li}$ atoms). In terms of the field operators $\hat{\Psi}_{\sigma}(\mathbf{r})$ the Hamiltonian of the system is given by

$$
\begin{aligned}
\hat{H}= & \sum_{\sigma=\uparrow, \downarrow} \int d \mathbf{r} \hat{\Psi}_{\sigma}^{\dagger}(\mathbf{r})\left(-\frac{\hbar^{2} \nabla^{2}}{2 m_{\sigma}}+V_{\sigma}(\mathbf{r})\right) \hat{\Psi}_{\sigma}(\mathbf{r}) \\
& +\iint d \mathbf{r} d \mathbf{r}^{\prime} \hat{\Psi}_{\uparrow}^{\dagger}(\mathbf{r}) \hat{\Psi}_{\downarrow}^{\dagger}\left(\mathbf{r}^{\prime}\right) g\left(\mathbf{r}, \mathbf{r}^{\prime}\right) \\
& \times \hat{\Psi}_{\downarrow}\left(\mathbf{r}^{\prime}\right) \hat{\Psi}_{\uparrow}(\mathbf{r})-\sum_{\sigma=\uparrow, \downarrow} \mu_{\sigma} \hat{N}_{\sigma} \\
= & \sum_{\sigma=\uparrow, \downarrow} \int d \mathbf{r} \hat{\Psi}_{\sigma}^{\dagger}(\mathbf{r})\left(-\frac{\hbar^{2} \nabla^{2}}{2 m_{\sigma}}+V_{\sigma}(\mathbf{r})-\mu_{\sigma}\right) \hat{\Psi}_{\sigma}(\mathbf{r}) \\
& +\iint d \mathbf{r} d \mathbf{r}^{\prime} \hat{\Psi}_{\uparrow}^{\dagger}(\mathbf{r}) \hat{\Psi}_{\downarrow}^{\dagger}\left(\mathbf{r}^{\prime}\right) g\left(\mathbf{r}, \mathbf{r}^{\prime}\right) \hat{\Psi}_{\downarrow}\left(\mathbf{r}^{\prime}\right) \hat{\Psi}_{\uparrow}(\mathbf{r}),
\end{aligned}
$$

where $\hbar=h /(2 \pi)$ and $h$ is the Planck constant. $\mu_{\sigma}$ is the chemical potential of the component $\sigma$ and the lattice potential is given by $V_{\sigma}(\mathbf{r})=s E_{r} \Sigma_{i=1}^{3} \sin ^{2}\left(k x_{j}\right)$, where $s$ is the lattice depth and $E_{r}=\hbar^{2} k^{2} /(2 m)$ is the recoil energy $(k=\pi / d$ and $d$ is the lattice constant). In the usual way the interaction between atoms is modeled by a contact potential

$$
g\left(\mathbf{r}, \mathbf{r}^{\prime}\right)=\frac{4 \pi \hbar^{2} a}{m} \delta\left(\mathbf{r}-\mathbf{r}^{\prime}\right),
$$

where $a$ is the $s$-wave scattering length. The number operator of the component $\sigma$,

$$
\hat{N}_{\sigma}=\int d \mathbf{r} \hat{n}_{\sigma}(\mathbf{r})=\int d \mathbf{r} \hat{\Psi}_{\sigma}^{\dagger}(\mathbf{r}) \hat{\Psi}_{\sigma}(\mathbf{r}),
$$

is expressed in terms of the density operator $\hat{n}_{\sigma}(\mathbf{r})$ $=\hat{\Psi}_{\sigma}^{\dagger}(\mathbf{r}) \hat{\Psi}_{\sigma}(\mathbf{r})$. In our case the field operators are fermionic, which implies fermionic equal time anticommutation relations

$$
\begin{gathered}
\left\{\hat{\Psi}_{\alpha}^{\dagger}(\mathbf{r}), \hat{\Psi}_{\beta}\left(\mathbf{r}^{\prime}\right)\right\}=\hat{\Psi}_{\alpha}^{\dagger}(\mathbf{r}) \hat{\Psi}_{\beta}\left(\mathbf{r}^{\prime}\right)+\hat{\Psi}_{\beta}\left(\mathbf{r}^{\prime}\right) \hat{\Psi}_{\alpha}^{\dagger}(\mathbf{r}) \\
=\delta_{\alpha \beta} \delta\left(\mathbf{r}-\mathbf{r}^{\prime}\right), \\
\left\{\hat{\Psi}_{\alpha}^{\dagger}(\mathbf{r}), \hat{\Psi}_{\beta}^{\dagger}\left(\mathbf{r}^{\prime}\right)\right\}=\left\{\hat{\Psi}_{\alpha}(\mathbf{r}), \hat{\Psi}_{\beta}\left(\mathbf{r}^{\prime}\right)\right\}=0,
\end{gathered}
$$

where $\delta_{\alpha \beta}$ is the Kronecker delta, and $\delta\left(\mathbf{r}-\mathbf{r}^{\prime}\right)$ is the Dirac delta function.

\section{A. Hubbard model}

In a sufficiently deep lattice we can expand the field operators in terms of the well localized (lowest band) Wannier functions as

$$
\hat{\Psi}_{\sigma}(\mathbf{r})=\sum_{i} w_{i}(\mathbf{r}) \hat{c}_{\sigma, i}
$$

where $w_{i}(\mathbf{r})$ is Wannier function centered around a lattice point $i$, and $\hat{c}_{\sigma, i}$ is a fermionic annihilation operator which annihilates fermions of component $\sigma$ at site $i$.

In assuming that only the lowest band states are occupied we are assuming temperatures which are much lower than the band gap. We can estimate the energy of the vibrational levels by approximating lattice wells with harmonic oscillators and in this way find that temperatures should satisfy a criterion

$$
k_{B} T \ll \frac{\hbar \pi}{d} \sqrt{\frac{2 s E_{r}}{m}},
$$

where $k_{B}$ is the Boltzmann constant. If, for concreteness, we assume ${ }^{6} \mathrm{Li}$ atoms in a cubic lattice with a lattice spacing 505 $\mathrm{nm}$, the above condition implies temperatures much below $5 \mu \mathrm{K}$ for a lattice depth of $10 E_{r}$.

It is assumed that only tunneling between the nearest neighbors is of importance. This assumption of tight binding is reasonable when the Wannier functions are well localized. In other words, overlap integrals between the next nearest neighbor Wannier functions are small compared to overlap integrals between the nearest neighbor Wannier functions. This always happens when the lattice is deep enough [36]. Using this approximation one finds the Hubbard Hamiltonian [37] 


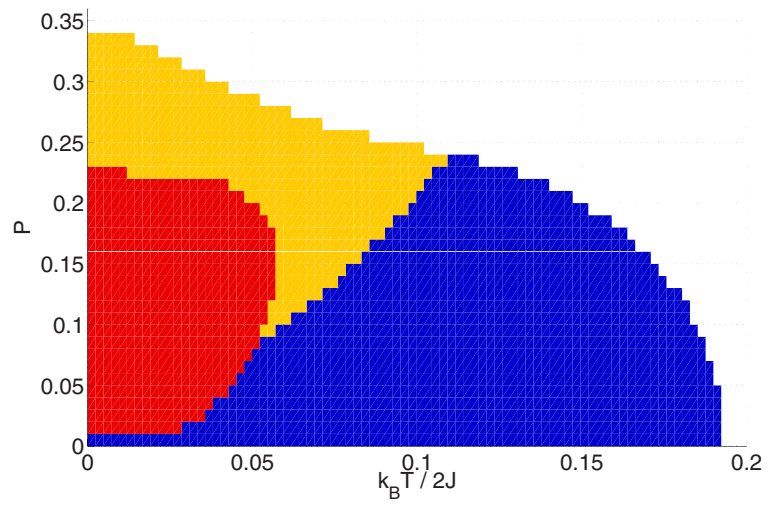

FIG. 1. (Color online) The phase diagram of Fermi gas in a 3D lattice [22]. Colors (or shading): BCS or BP states=blue or dark gray, $\mathrm{FFLO}=$ yellow or light gray, phase separation=red or gray, and normal gas $=$ white. The parameters were such that the average filling fraction $\left(n_{\uparrow}+n_{\downarrow}\right) / 2=0.2, J=0.07 E_{r}$, and $U /(2 J)=-1.86$.

$$
\begin{aligned}
\hat{H}= & \sum_{n}\left(-\mu_{\uparrow} \hat{c}_{\uparrow, n}^{\dagger} \hat{c}_{\uparrow, n}-\mu_{\downarrow} \hat{c}_{\downarrow, n}^{\dagger} \hat{c}_{\downarrow, n}\right)+U \sum_{n} \hat{c}_{\uparrow, n}^{\dagger} \hat{c}_{\downarrow, n}^{\dagger} \hat{c}_{\downarrow, n} \hat{c}_{\uparrow, n} \\
& -\left(J_{x} \sum_{\langle n, m\rangle_{x}}+J_{y} \sum_{\langle n, m\rangle_{y}}+J_{z} \sum_{\langle n, m\rangle_{z}}\right)\left(\hat{c}_{\uparrow, m}^{\dagger} \hat{c}_{\uparrow, n}+\hat{c}_{\downarrow, n}^{\dagger} \hat{c}_{\downarrow, n}\right),
\end{aligned}
$$

where $\langle m, n\rangle_{\alpha}$ means a sum over the nearest neighbors in the $\alpha$ direction and hopping strength is defined by

$$
J_{\alpha}=-\int d \mathbf{r} w_{n}^{*}(\mathbf{r})\left(-\frac{\hbar^{2} \nabla^{2}}{2 m}+s E_{r} \sum_{i} \sin ^{2}\left(k x_{i}\right)\right) w_{n \pm d \hat{x}_{\alpha}}(\mathbf{r}) .
$$

Furthermore, the dominant on site interaction coupling strength is given by

$$
U=\frac{4 \pi \hbar^{2} a}{m} \int d \mathbf{r}\left|w_{i}(\mathbf{r})\right|^{4}
$$

In our calculations we have approximated the Wannier functions with a harmonic oscillator ground state in each potential well.

The most stringent criteria for temperatures follows from the fact that critical temperature for the superfluidity is typically much less than the Fermi energy, which in turn is less than the bandwidth. This means that in the regime of greatest interest, the temperature should be of same order or less than the tunneling energy scale $J$.

\section{B. Ground state ansatz at zero temperature}

On a mean-field level in the homogeneous system the ground state ansatz at zero temperature, which includes the possibility of the BCS state [38], the breach pair (BP) state $[9,10]$, and the plane wave FFLO state $[7,8]$ can be expressed as [39]

$$
\left|\Psi_{G S}\right\rangle=\prod_{\mathbf{k} \in G_{3}} \hat{c}_{\downarrow,-\mathbf{k}+\mathbf{q}}^{\dagger} \prod_{\mathbf{k} \in G_{2}} \hat{c}_{\uparrow, \mathbf{k}}^{\dagger} \prod_{\mathbf{k} \in G_{1}}\left(u_{\mathbf{k}, \mathbf{q}}+v_{\mathbf{k}, \mathbf{q}} \hat{c}_{\uparrow, \mathbf{k}}^{\dagger} \hat{c}_{\downarrow,-\mathbf{k}+\mathbf{q}}^{\dagger}\right)|0\rangle,
$$

where $|0\rangle$ is the vacuum state. In area $G_{1}$ both quasiparticle dispersions $E_{\uparrow, \mathbf{k}, \mathbf{q}}, E_{\downarrow, \mathbf{k}, \mathbf{q}}$ are positive, in area $G_{2} E_{\downarrow, \mathbf{k}, \mathbf{q}} \geq 0$ and $E_{\uparrow, \mathbf{k}, \mathbf{q}}<0$, and in area $G_{3} E_{\uparrow, \mathbf{k}, \mathbf{q}} \geq 0$ and $E_{\downarrow, \mathbf{k}, \mathbf{q}}<0$. Area $G_{1}$ is populated by pairs with a quasimomentum q. Area $G_{2}$ is populated by atoms of the component $\uparrow$ in the quasimomentum states $\mathbf{k} \in G_{2}$ while the area $G_{3}$ is populated by $\downarrow$ atoms in the quasimomentum states $-\mathbf{k}+\mathbf{q}$. By diagonalizing the mean-field theory one finds the quasiparticle dispersions

$$
E_{\downarrow / \uparrow, \mathbf{k}, \mathbf{q}}=\sqrt{\left(\frac{\epsilon_{\uparrow, \mathbf{k}}+\epsilon_{\downarrow,-\mathbf{k}+\mathbf{q}}}{2}\right)^{2}+\Delta^{2}} \mp \frac{\epsilon_{\uparrow, \mathbf{k}}-\epsilon_{\downarrow,-\mathbf{k}+\mathbf{q}}}{2},
$$

where $\Delta$ is the pairing gap and the single particle dispersions are

$$
\epsilon_{\sigma, \mathbf{k}}=\sum_{\alpha=1}^{3} 2 J_{\alpha}\left(1-\cos \left(k_{\alpha} d\right)\right)-\mu_{\sigma} .
$$

The quasiparticle amplitudes $u_{\mathbf{k}, \mathbf{q}}$ and $v_{\mathbf{k}, \mathbf{q}}$ are given by

$$
\begin{gathered}
u_{\mathbf{k}, \mathbf{q}}^{2}=\frac{1}{2}\left(1+\frac{\epsilon_{\uparrow, \mathbf{k}}+\boldsymbol{\epsilon}_{\downarrow,-\mathbf{k}+\mathbf{q}}}{2 \sqrt{\left[\left(\epsilon_{\uparrow, \mathbf{k}}+\epsilon_{\downarrow,-\mathbf{k}+\mathbf{q}}\right) / 2\right]^{2}+\Delta^{2}}}\right), \\
v_{\mathbf{k}, \mathbf{q}}^{2}=1-u_{\mathbf{k}, \mathbf{q}}^{2} .
\end{gathered}
$$

Based on the properties of this ansatz, it can be classified further. When the wave vector $\mathbf{q} \neq 0$, the ground state is called the FFLO state, which breaks the translational symmetry. If $\mathbf{q}=0$ and both quasiparticle dispersions $E_{\uparrow, \mathbf{k}}, E_{\downarrow, \mathbf{k}}$ are positive for all $\mathbf{k}$ vectors, the ground state is called the BCS state, and finally, if $\mathbf{q}=0$ and $E_{\sigma, \mathbf{k}}<0$ for some $\mathbf{k}$ vectors, the ground state is called the BP state. This last case involves phase separation in the momentum space.

In Fig. 1 we show an example phase diagram of the polarized system in a three-dimensional lattice [22]. At low temperatures, phase separation is favored for low polarization, while for larger polarization the FFLO state is energetically favorable in substantially large parts of the phase diagram. At nonzero temperatures the BP state can be energetically stable and occupy regions of the phase diagram close to the critical temperature. Although we do not show it in the figure, for low polarization quasiparticle dispersions of the BP state can be gapped while for higher polarization they are gapless. Very close to the critical temperature the dispersions are always gapless for the polarized system. Note that only BCS-normal phase separation was considered, not BCS-FFLO coexistence, which could make the parameter window where FFLO occurs even wider.

\section{NOISE CORRELATIONS}

Our aim is to compute the noise correlation functions which are defined by [27]

$$
\begin{aligned}
G_{\alpha \beta}\left(\mathbf{r}, \mathbf{r}^{\prime}\right)= & \left\langle\hat{n}_{\alpha}(\mathbf{r}, t) \hat{n}_{\beta}\left(\mathbf{r}^{\prime}, t\right)\right\rangle-\left\langle\hat{n}_{\alpha}(\mathbf{r}, t)\right\rangle\left\langle\hat{n}_{\beta}\left(\mathbf{r}^{\prime}, t\right)\right\rangle \\
= & \left\langle\hat{\Psi}_{\alpha}^{\dagger}(\mathbf{r}, t) \hat{\Psi}_{\beta}^{\dagger}\left(\mathbf{r}^{\prime}, t\right) \hat{\Psi}_{\beta}\left(\mathbf{r}^{\prime}, t\right) \hat{\Psi}_{\alpha}(\mathbf{r}, t)\right\rangle \\
& +\delta_{\alpha \beta} \delta\left(\mathbf{r}-\mathbf{r}^{\prime}\right)\left\langle\hat{n}_{\alpha}(\mathbf{r}, t)\right\rangle-\left\langle\hat{n}_{\alpha}(\mathbf{r}, t)\right\rangle\left\langle\hat{n}_{\beta}\left(\mathbf{r}^{\prime}, t\right)\right\rangle,
\end{aligned}
$$

where $\alpha$ and $\beta$ are component indices, $\mathbf{r}$ and $\mathbf{r}^{\prime}$ are the positions, and $t$ is time. The term with Dirac's delta function is 
due to the normal ordering of the density-density expectation value.

Often in experiments a gas which has been trapped is released and the gas expands. When collisions during the expansion can be ignored and the expansion is ballistic, the density-density correlations after the expansion reflect correlations in momentum space at $t=0$. Therefore, correlations can be computed using the wave function in momentum space prior to expansion. Positions in real space after time $t$ are related to $k$ vectors through $\mathbf{r}=t \mathbf{k} / \mathrm{m}$. In other words at long times (the saddle point approximation of the free evolution amounts to the limit $\left.t \gg m d^{2} / \hbar\right)$,

$$
w_{i}(\mathbf{r}, t) \sim e^{-i \mathbf{Q}(\mathbf{r}) \cdot \mathbf{R}_{i}},
$$

where $\mathbf{R}_{i}=d\left(i_{x}, i_{y}, i_{z}\right)\left(i_{n}\right.$ are integers $)$ is a lattice vector and

$$
\mathbf{Q}(\mathbf{r})=\frac{m \mathbf{r}}{\hbar t}
$$

Thus the densities become

$$
n_{\alpha}(\mathbf{r}, t)=\left\langle\hat{n}_{\alpha}(\mathbf{r}, t)\right\rangle=A(t)^{2} \sum_{i, j} e^{i \mathbf{Q}(\mathbf{r}) \cdot \mathbf{R}_{i j}}\left\langle\hat{c}_{\alpha, i}^{\dagger} \hat{c}_{\alpha, j}\right\rangle,
$$

where $\mathbf{R}_{i j}=\mathbf{R}_{i}-\mathbf{R}_{j}$ and $A(t)$ is a time dependent scale factor which depends on the Wannier functions. The correlation functions in Eq. (9) now become

$$
\begin{aligned}
G_{\alpha \beta}\left(\mathbf{r}, \mathbf{r}^{\prime}\right)= & A(t)^{4} \sum_{i, j, m, n} e^{i \mathbf{Q}(\mathbf{r}) \cdot \mathbf{R}_{i j}+i \mathbf{Q}\left(\mathbf{r}^{\prime}\right) \cdot \mathbf{R}_{m n}}\left\langle\hat{c}_{\alpha, i}^{\dagger} \hat{c}_{\beta, m}^{\dagger} \hat{c}_{\beta, n} \hat{c}_{\alpha, j}\right\rangle \\
& +\delta_{\alpha \beta} \delta\left(\mathbf{r}-\mathbf{r}^{\prime}\right)\left\langle\hat{n}_{\alpha}(\mathbf{r}, t)\right\rangle-\left\langle\hat{n}_{\alpha}(\mathbf{r}, t)\right\rangle\left\langle\hat{n}_{\beta}\left(\mathbf{r}^{\prime}, t\right)\right\rangle .
\end{aligned}
$$

\section{A. Noise correlations of the BCS state at zero temperature}

We review here the noise correlations of the BCS state. The usual unpolarized BCS ground state is extracted from the ansatz in Eq. (6) by setting both chemical potentials to be equal and $\mathbf{q}=\mathbf{0}$. For the BCS state both quasiparticle dispersions are positive. Because the BCS ground state is presented in momentum space, for simplicity we express the densities in Eq. (10) in terms of the operators in the momentum space

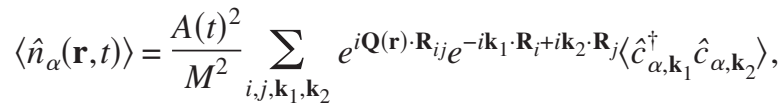

and similarly for the noise correlations in Eq. (11),

$$
\begin{aligned}
G_{\alpha \beta}\left(\mathbf{r}, \mathbf{r}^{\prime}\right)= & \frac{A(t)^{4}}{M^{4}} \sum_{\substack{i, j, m, n, \mathbf{k}_{1}, \mathbf{k}_{2}, \mathbf{k}_{3}, \mathbf{k}_{4}}}\left(e^{i \mathbf{Q}(\mathbf{r}) \cdot \mathbf{R}_{i j}+i \mathbf{Q}\left(\mathbf{r}^{\prime}\right) \cdot \mathbf{R}_{m n}} e^{\left.-i \mathbf{k}_{1} \cdot \mathbf{R}_{i}+i \mathbf{k}_{2} \cdot \mathbf{R}_{j}-i \mathbf{k}_{3} \cdot \mathbf{R}_{m}+i \mathbf{k}_{4} \cdot \mathbf{R}_{n}\left\langle\hat{c}_{\alpha, \mathbf{k}_{1}}^{\dagger} \hat{c}_{\beta, \mathbf{k}_{3}}^{\dagger} \hat{c}_{\beta, \mathbf{k}_{4}} \hat{c}_{\alpha, \mathbf{k}_{2}}\right\rangle\right)}\right. \\
& +\delta_{\alpha \beta} \delta\left(\mathbf{r}-\mathbf{r}^{\prime}\right)\left\langle\hat{n}_{\alpha}(\mathbf{r}, t)\right\rangle-\left\langle\hat{n}_{\alpha}(\mathbf{r}, t)\right\rangle\left\langle\hat{n}_{\beta}\left(\mathbf{r}^{\prime}, t\right)\right\rangle,
\end{aligned}
$$

where $M$ is the number of lattice sites. Now one needs the expectation values $\left\langle\hat{c}_{\alpha, \mathbf{k}_{1}}^{\dagger} \hat{c}_{\beta, \mathbf{k}_{3}}^{\dagger} \hat{c}_{\beta, \mathbf{k}_{4}} \hat{c}_{\alpha, \mathbf{k}_{2}}\right\rangle$ and $\left\langle\hat{c}_{\alpha, \mathbf{k}_{1}}^{\dagger} \hat{c}_{\alpha, \mathbf{k}_{2}}\right\rangle$. For the latter term one gets

$$
\left\langle\Psi_{B C S}\left|\hat{c}_{\alpha, \mathbf{k}_{1}}^{\dagger} \hat{c}_{\alpha, \mathbf{k}_{2}}\right| \Psi_{B C S}\right\rangle=\delta_{\mathbf{k}_{1} \mathbf{k}_{2}}\left|v_{\mathbf{k}_{1}}\right|^{2},
$$

and using this the densities in Eq. (12) can be expressed as

$$
\left\langle\hat{n}_{\alpha}(\mathbf{r}, t)\right\rangle=\frac{A(t)^{2}}{M^{2}} \sum_{i, j, \mathbf{k}} e^{i[\mathbf{Q}(\mathbf{r})-\mathbf{k}] \cdot \mathbf{R}_{i j}}\left|v_{\mathbf{k}}\right|^{2} .
$$

Now the number of particles is the same for both the components as it should be. When the lattice is large, i.e., when the lattice size is $M \gg 1$, the exponential terms only add constructively when $\mathbf{Q}(\mathbf{r})-\mathbf{k}=0$ and we can approximate the densities as

$$
\left\langle\hat{n}_{\alpha}(\mathbf{r}, t)\right\rangle=A(t)^{2} \sum_{\mathbf{k}} \delta\left(\mathbf{r}-\frac{\hbar t}{m} \mathbf{k}\right)\left|v_{\mathbf{k}}\right|^{2} .
$$

Nonvanishing four operator expectation values are

$$
\begin{gathered}
\left\langle\Psi_{B C S}\left|\hat{c}_{\uparrow, \mathbf{k}}^{\dagger} \hat{c}_{\downarrow,-\mathbf{k}^{\prime}}^{\dagger} \hat{c}_{\downarrow,-\mathbf{k}^{\prime}} \hat{c}_{\uparrow, \mathbf{k}}\right| \Psi_{B C S}\right\rangle \\
=\delta_{\mathbf{k} \mathbf{k}^{\prime}}\left|v_{\mathbf{k}}\right|^{2}+\left(1-\delta_{\mathbf{k k}^{\prime}}\right)\left|v_{\mathbf{k}^{\prime}}\right|^{2}\left|v_{\mathbf{k}}\right|^{2}, \\
\left\langle\Psi_{B C S}\left|\hat{c}_{\uparrow(\downarrow), \mathbf{k}}^{\dagger} \hat{c}_{\uparrow(\downarrow), \mathbf{k}^{\prime}}^{\dagger}, \hat{c}_{\uparrow(\downarrow), \mathbf{k}^{\prime}} \hat{c}_{\uparrow(\downarrow), \mathbf{k}}\right| \Psi_{B C S}\right\rangle=\left(1-\delta_{\mathbf{k k}^{\prime}}\right)\left|v_{\mathbf{k}^{\prime}}\right|^{2}\left|v_{\mathbf{k}}\right|^{2},
\end{gathered}
$$

and combining these results and taking the limit of large lattices, we find

$$
\begin{aligned}
G_{\uparrow \downarrow}\left(\mathbf{r}, \mathbf{r}^{\prime}\right)= & G_{\downarrow \uparrow}\left(\mathbf{r}, \mathbf{r}^{\prime}\right) \\
= & A(t) \sum_{\mathbf{k}}\left|u_{\mathbf{k}}\right|^{2}\left|v_{\mathbf{k}}\right|^{2} \delta\left(\mathbf{r}-\frac{\hbar t \tilde{\mathbf{k}}}{m}\right) \delta\left(\mathbf{r}^{\prime}+\frac{\hbar t \tilde{\mathbf{k}^{\prime}}}{m}\right), \\
G_{\sigma \sigma}\left(\mathbf{r}, \mathbf{r}^{\prime}\right)= & -A(t)^{4} \sum_{\mathbf{k}}\left|v_{\mathbf{k}}\right|^{4} \delta\left(\mathbf{r}-\frac{\hbar t \tilde{\mathbf{k}}}{m}\right) \delta\left(\mathbf{r}^{\prime}-\frac{\hbar t \tilde{\mathbf{k}}^{\prime}}{m}\right) \\
& +\delta\left(\mathbf{r}-\mathbf{r}^{\prime}\right)\left\langle\hat{n}_{\sigma}(\mathbf{r}, t)\right\rangle,
\end{aligned}
$$

where $\tilde{\mathbf{k}}=\mathbf{k}+\sum_{\alpha=1}^{3} 2 n_{\alpha} \pi \hat{x}_{\alpha} / d \quad$ and $\quad \tilde{\mathbf{k}}^{\prime}=\mathbf{k}+\sum_{\alpha=1}^{3} 2 m_{\alpha} \pi \hat{x}_{\alpha} / d$, 
where $\mathbf{k}$ is a lattice momentum, $\hat{x}_{\alpha}$ are orthogonal unit vectors, and $n_{i}$ and $m_{i}$ are integers. Because $\left|u_{\mathbf{k}}\right|^{2}\left|v_{\mathbf{k}}\right|^{2} \sim \Delta^{2}$ the noise correlation $G_{\uparrow \downarrow}$ between different components vanishes with vanishing gap $\Delta$ and is thus identically zero in the normal state. One can see that the noise correlations in the BCS state differ crucially from this. Because in the BCS state momenta $\mathbf{k}$ and $-\mathbf{k}$ are correlated, the noise correlation signal is pronounced only if

$$
\mathbf{r}+\mathbf{r}^{\prime}=\sum_{i=1}^{3} \frac{\hbar 2 n_{i} \pi t \hat{x}_{i}}{m d}
$$

The continuum result [27] is similar, but peaks appear when $\mathbf{r}+\mathbf{r}^{\prime}=0$. In the continuum limit our result reduces to the known result.

On the other hand, correlations of the single component $G_{\uparrow \uparrow}$ and $G_{\downarrow \downarrow}$ show a hole when

$$
\mathbf{r}-\mathbf{r}^{\prime}=\sum_{i=1}^{3} \frac{2 \hbar n_{i} \pi t \hat{x}_{i}}{m d}
$$

and $n_{i} \neq 0$ for at least one $i$ [40]. The reason for this is the fermionic antibunching related to Pauli blocking, i.e., two or more identical fermions cannot be in the same momentum state. This fermionic antibunching was also measured in the noise correlations of an ideal Fermi gas [29].

In Fig. 2 we demonstrate the BCS noise correlations at $T=0$. In Fig. 2(a) we show a cut in the $z=0$ plane of the BCS noise correlation between the components while in Fig. 2(b) we plot the column integrated BCS-state noise correlations

$$
\begin{aligned}
G_{\uparrow, \downarrow}(x, y)= & \int d z G_{\uparrow, \downarrow}\left(x, y, z,-x+\hbar t q_{x} / m,-y\right. \\
& \left.+\hbar t q_{y} / m,-z+\hbar t q_{z} / m\right) .
\end{aligned}
$$

As one can see from Fig. 2, the noise correlations reflect underlying lattice structure and strongly nonspherical structure of the Fermi surface. We can see also from Figs. 2(a) and 2(b) that the peaks are highest near the Fermi surfaces where $\epsilon_{\uparrow, \mathbf{k}}+\epsilon_{\downarrow,-\mathbf{k}}=0$. In the weakly interacting BCS limit correlations are strongly peaked at the Fermi surface, but the distribution of the peak heights becomes broad in the BEC limit, and the signal approaches the interference signal of the Bose-Einstein condensate.

In Fig. 2(c) we show the BCS-state correlations of a single component in the $z=0$ plane. As is clear, the BCS-state noise correlation of a single component shows similar antibunching behavior as the ideal Fermi gas noise correlation. If $\mathbf{r}=\mathbf{r}^{\prime}+\sum_{i=1}^{3} 2 \hbar n_{i} \pi t \hat{x}_{i} /(m d)$ and $n_{i} \neq 0$ for at least one $i$, then the noise correlation of a single component shows the holes. However, the BCS-state noise correlation of a single component does also show the bunching peak when $\mathbf{r}=\mathbf{r}^{\prime}$. This result differs from the ideal gas result where such a peak is absent.

\section{B. Noise correlations of the BP state at zero temperature}

The ground state in Eq. (6) is the BP state when $\mathbf{q}=0$ and one of the quasiparticle dispersions $E_{\sigma, \mathbf{k}}$ is negative for some (a)

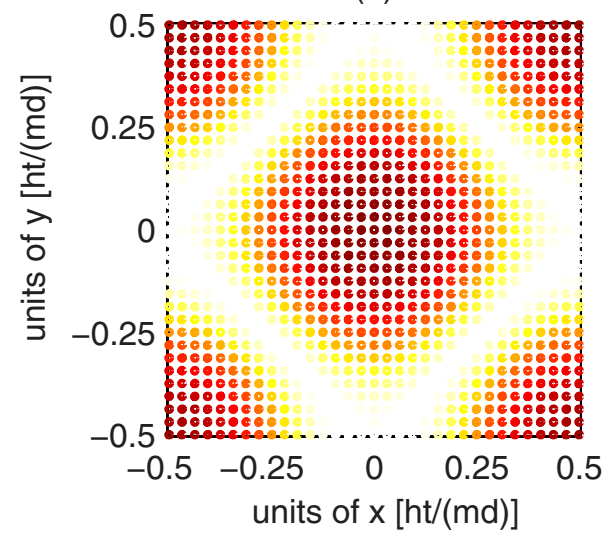

(b)

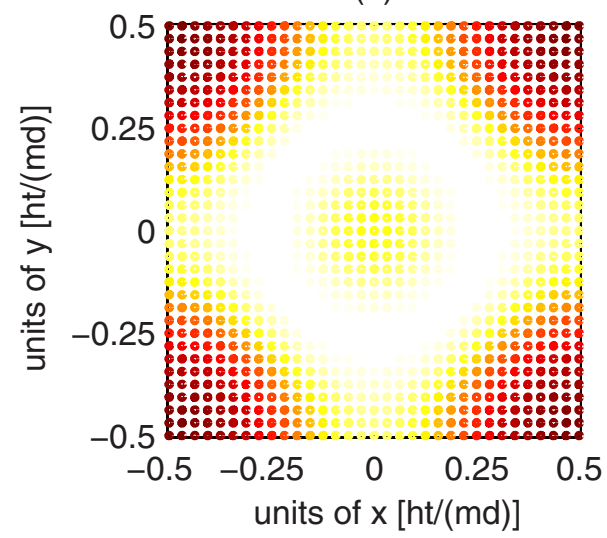

(c)

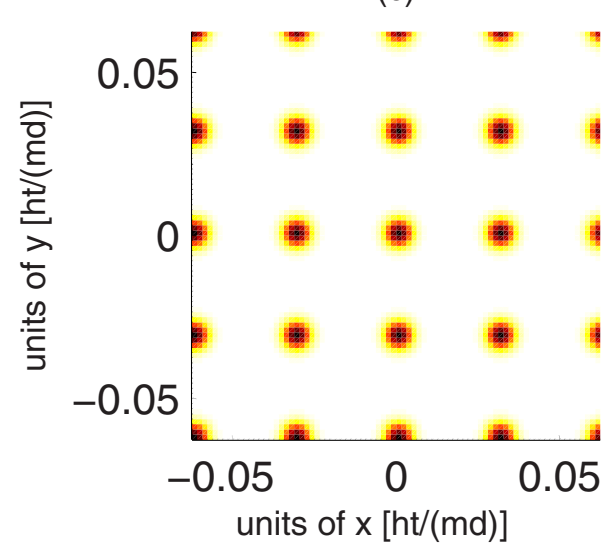

FIG. 2. (Color online) In (a) we show a cut of the BCS noise correlations between components in the $z=0$ plane. In (b) we show the integrated BCS correlations between the components. In (c) we demonstrate the noise correlation of a single component in the plane $z=0$. As parameters we used the average filling fraction $\left(n_{\uparrow}\right.$ $\left.+n_{\downarrow}\right) / 2=0.30$, polarization $P=\left(n_{\uparrow}-n_{\downarrow}\right) /\left(n_{\uparrow}+n_{\downarrow}\right)=0.0, \quad \Delta /(2 J)$ $=1.03$, and $U /(2 J)=-3.0$. All these examples are calculated at $T$ $=0$. In (a) and (b) we choose $\mathbf{r}^{\prime}=-\mathbf{r}$ and in (c) we choose $y^{\prime}=y$, $z=z^{\prime}=0$, and $x^{\prime}=x+\hbar t 2 \pi /(m d)$. Color coding is such that light colors imply high peaks and dark colors imply low peaks.

values of the momentum $\mathbf{k}$. This implies that in the BP state $|\delta \mu|=\left|\mu_{\uparrow}-\mu_{\downarrow}\right|>2 \Delta$. The BP state is a gapless state, because one of the quasiparticle dispersions changes its sign when the 
momentum varies. The BP state at zero temperature is expressed as

$$
\left|\Psi_{B P}\right\rangle=\prod_{\mathbf{k} \in G_{3}} \hat{c}_{\downarrow,-\mathbf{k}}^{\dagger} \prod_{\mathbf{k} \in G_{2}} \hat{c}_{\uparrow, \mathbf{k}}^{\dagger} \prod_{\mathbf{k} \in G_{1}}\left(u_{\mathbf{k}}+v_{\mathbf{k}} \hat{c}_{\uparrow, \mathbf{k}}^{\dagger} \hat{c}_{\downarrow,-\mathbf{k}}^{\dagger}\right)|0\rangle .
$$

In the area $G_{1}$ both quasiparticle dispersions $E_{\uparrow, \mathbf{k}, \mathbf{q}}, E_{\downarrow, \mathbf{k}, \mathbf{q}}$ $\geq 0$, in the area $G_{2} E_{\downarrow, \mathbf{k}} \geq 0$ and $E_{\uparrow, \mathbf{k}}<0$, and in the area $G_{3}$ $E_{\uparrow, \mathbf{k}} \geq 0$ and $E_{\downarrow, \mathbf{k}}<0$.
Now we can calculate the required expectation values similarly as before. We find that the densities in the BP state (in a large lattice) are

$$
\begin{aligned}
\left\langle\hat{n}_{\uparrow(\downarrow)}(\mathbf{r}, t)\right\rangle= & A(t)^{2} \sum_{\mathbf{k}} \delta\left(\mathbf{r}-\frac{\hbar t \mathbf{k}}{m}\right)\left[1-\theta\left(E_{\uparrow(\downarrow), \mathbf{k}}\right)\right. \\
& \left.+\theta\left(E_{\uparrow, \mathbf{k}}\right) \theta\left(E_{\downarrow, \mathbf{k}}\right)\left|v_{\mathbf{k}}\right|^{2}\right],
\end{aligned}
$$

and nonvanishing expectation values in the noise correlations in Eq. (13) are in turn given by

$$
\begin{aligned}
\left\langle\Psi_{B P}\left|\hat{c}_{\uparrow, \mathbf{k}^{\dagger}}^{\dagger} \hat{c}_{\downarrow,-\mathbf{k}^{\prime}}^{\dagger} \hat{c}_{\downarrow,-\mathbf{k}^{\prime}} \hat{c}_{\uparrow, \mathbf{k}}\right| \Psi_{B P}\right\rangle= & \left(1-\delta_{\mathbf{k} \mathbf{k}^{\prime}}\right)\left[\left(1-\theta\left(E_{\uparrow, \mathbf{k}}\right)\right)\left(1-\theta\left(E_{\downarrow, \mathbf{k}^{\prime}}\right)\right)+\theta\left(E_{\uparrow, \mathbf{k}}\right) \theta\left(E_{\downarrow, \mathbf{k}}\right) \theta\left(E_{\uparrow, \mathbf{k}^{\prime}}\right) \theta\left(E_{\downarrow, \mathbf{k}^{\prime}}\right)\left|v_{\mathbf{k}}\right|^{2}\left|v_{\mathbf{k}^{\prime}}\right|^{2}\right. \\
& \left.+\theta\left(E_{\uparrow, \mathbf{k}}\right) \theta\left(E_{\downarrow, \mathbf{k}}\right)\left(1-\theta\left(E_{\downarrow, \mathbf{k}^{\prime}}\right)\right)\left|v_{\mathbf{k}}\right|^{2}+\theta\left(E_{\uparrow, \mathbf{k}^{\prime}}\right) \theta\left(E_{\downarrow, \mathbf{k}^{\prime}}\right)\left(1-\theta\left(E_{\uparrow, \mathbf{k}}\right)\right)\left|v_{\mathbf{k}^{\prime}}\right|^{2}\right] \\
& +\delta_{\mathbf{k} \mathbf{k}^{\prime}} \theta\left(E_{\uparrow, \mathbf{k}}\right) \theta\left(E_{\downarrow, \mathbf{k}}\right)\left|v_{\mathbf{k}}\right|^{2}\left\langle\Psi_{B P}\left|\hat{c}_{\uparrow(\downarrow), \mathbf{k}}^{\dagger} \hat{c}_{\uparrow(\downarrow), \mathbf{q}}^{\dagger} \hat{c}_{\uparrow(\downarrow), \mathbf{k}^{\prime}} \hat{c}_{\uparrow(\downarrow), \mathbf{k}}\right| \Psi_{B P}\right\rangle \\
= & \left(1-\delta_{\mathbf{k} \mathbf{k}^{\prime}}\right)\left[\left(1-\theta\left(E_{\uparrow(\downarrow), \mathbf{k}}\right)\right)\left(1-\theta\left(E_{\uparrow(\downarrow), \mathbf{k}^{\prime}}\right)\right)+\theta\left(E_{\uparrow, \mathbf{k}}\right) \theta\left(E_{\downarrow, \mathbf{k}}\right) \theta\left(E_{\uparrow, \mathbf{q}^{\prime}}\right) \theta\left(E_{\downarrow, \mathbf{k}^{\prime}}\right)\left|v_{\mathbf{k}}\right|^{2}\left|v_{\mathbf{k}^{\prime}}\right|^{2}\right. \\
& \left.+\theta\left(E_{\uparrow, \mathbf{k}}\right) \theta\left(E_{\downarrow, \mathbf{k}}\right)\left(1-\theta\left(E_{\uparrow(\downarrow), \mathbf{k}^{\prime}}\right)\right)\left|v_{\mathbf{k}}\right|^{2}+\theta\left(E_{\uparrow, \mathbf{k}^{\prime}}\right) \theta\left(E_{\downarrow, \mathbf{k}^{\prime}}\right)\left(1-\theta\left(E_{\uparrow,(\downarrow) \mathbf{k}}\right)\right)\left|v_{\mathbf{k}^{\prime}}\right|^{2}\right] .
\end{aligned}
$$

Subtracting the densities cancels out most of these terms and again for large lattices we get strongly peaked behavior and

$$
\begin{aligned}
G_{\uparrow \downarrow(\downarrow \uparrow)}\left(\mathbf{r}, \mathbf{r}^{\prime}\right)= & A(t)^{4} \sum_{\mathbf{k}} \delta\left(\mathbf{r}-\frac{\hbar t \tilde{\mathbf{k}}}{m}\right) \\
& \times \delta\left(\mathbf{r}^{\prime}+\frac{\hbar t \tilde{\mathbf{k}}^{\prime}}{m}\right) \theta\left(E_{\uparrow, \mathbf{k}}\right) \theta\left(E_{\downarrow, \mathbf{k}}\right)\left|u_{\mathbf{k}}\right|^{2}\left|v_{\mathbf{k}}\right|^{2}, \\
G_{\sigma \sigma}\left(\mathbf{r}, \mathbf{r}^{\prime}\right)= & -A(t)^{4} \sum_{\mathbf{k}}\left\{\delta\left(\mathbf{r}-\frac{\hbar t \tilde{\mathbf{k}}}{m}\right)\right. \\
& \times \delta\left(\mathbf{r}^{\prime}-\frac{\hbar t \tilde{\mathbf{k}}^{\prime}}{m}\right) \theta\left(E_{\uparrow, \mathbf{k}}\right) \theta\left(E_{\downarrow, \mathbf{k}}\right)\left|v_{\mathbf{k}}\right|^{4} \\
& \left.+\left[1-\theta\left(E_{\sigma, \mathbf{k}}\right)\right]\right\}+\delta\left(\mathbf{r}-\mathbf{r}^{\prime}\right)\left\langle\hat{n}_{\sigma}(\mathbf{r}, t)\right\rangle . \quad
\end{aligned}
$$

In the correlation between different components there are now areas where peaks are absent. The reason for this is that the BP state has phase separation in the momentum space. Because in the BP state there is no pairing between momenta $\mathbf{k}$ and $-\mathbf{k}$, if $E_{\uparrow, \mathbf{k}}<0$ or $E_{\downarrow, \mathbf{k}}<0$, in contrast to the BCS state, there are no correlations between the points

$$
\mathbf{r}+\mathbf{r}^{\prime}=\sum_{i=1}^{3} \frac{\hbar 2 n_{i} \pi t \hat{x}_{i}}{m d}
$$

in this region.

In Fig. 3 we demonstrate the BP-state noise correlations at $T=0$. In Fig. 3(a) we have plotted the BP-state noise correlation at the $z=0$ plane while in Fig. 3(b) we show the corresponding column integrated noise correlation. From Fig. 3(a) one can see clear areas without correlation peaks in the $\mathrm{BP}$ noise correlation due to the fact that these areas are populated only by atoms of the majority component which are unpaired. From the integrated correlations in Fig. 3(b) we can see that the peaks are now relatively low in the area between the Fermi surfaces. This differs substantially from the integrated BCS noise correlation [see Fig. 2(b)].

Results in this section are intended mainly as a reference since the BP state is not expected to be the ground state at zero temperature. Depending on polarization the ground state at zero temperature can be the BCS state, phase separation [41], or the FFLO-type state [22]. However, at nonzero temperatures the $\mathrm{BP}$ state can be the minimum of the free energy [42].

\section{Noise correlations of the plane wave FFLO state at zero temperature}

The FFLO ground state is presented in Eq. (6). Contrary to the BCS state and the BP state, in the FFLO state the parameter $\mathbf{q} \neq 0$ and the translational invariance is broken. In this case the order parameter is given by $\Delta\left(\mathbf{R}_{i}\right)$ $=\Delta_{0} \exp \left(i \mathbf{q} \cdot \mathbf{R}_{i}\right)$, where $\mathbf{R}_{i}$ is a lattice vector. In the FFLO state the other Fermi surface has been effectively shifted by the wave vector $\mathbf{q}$.

The densities in the FFLO state are (in the limit of large lattices) given by

$$
\begin{aligned}
\left\langle\hat{n}_{\uparrow}(\mathbf{r}, t)\right\rangle= & A(t)^{2} \sum_{\mathbf{k}} \delta\left(\mathbf{r}-\frac{\hbar t \mathbf{k}}{m}\right) \\
& \times\left[1-\theta\left(E_{\uparrow, \mathbf{k}, \mathbf{q}}\right)+\theta\left(E_{\uparrow, \mathbf{k}, \mathbf{q}}\right) \theta\left(E_{\downarrow, \mathbf{k}, \mathbf{q}}\right)\left|v_{\mathbf{k}, \mathbf{q}}\right|^{2}\right],
\end{aligned}
$$


(a)

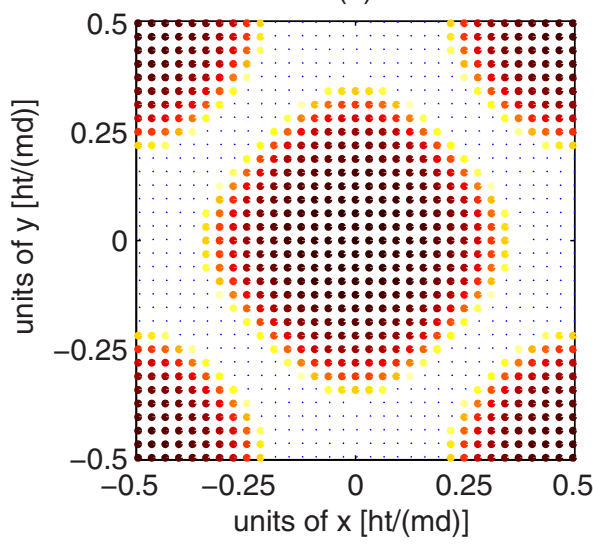

(b)

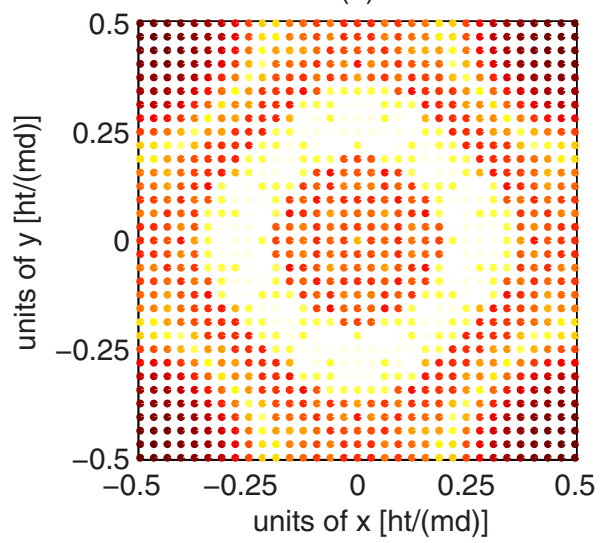

FIG. 3. (Color online) In this figure we demonstrate examples of the BP noise correlations between the components at $T=0$. In (a) we demonstrate a cut in the $z=0$ plane while in (b) we show the column integrated BP noise correlation. Parameters we used were $\Delta /(2 J)$ $=0.318,\left(\mu_{\uparrow}+\mu_{\downarrow}\right) / 2.0=2.18$, and $\delta \mu=\mu_{\uparrow}-\mu_{\downarrow}=1.14$. In (a) the correlations vanish in the dotted area. We have chosen $\mathbf{r}^{\prime}=-\mathbf{r}$ and color coding is such that light colors imply high peaks and dark colors imply low peaks.

$$
\begin{aligned}
\left\langle\hat{n}_{\downarrow}(\mathbf{r}, t)\right\rangle= & A(t)^{2} \sum_{\mathbf{k}} \delta\left(\mathbf{r}-\frac{\hbar t(\mathbf{q}-\mathbf{k})}{m}\right) \\
& \times\left[1-\theta\left(E_{\downarrow, \mathbf{k}, \mathbf{q}}\right)+\theta\left(E_{\uparrow, \mathbf{k}, \mathbf{q}}\right) \theta\left(E_{\downarrow, \mathbf{k}, \mathbf{q}}\right)\left|v_{\mathbf{k}, \mathbf{q}}\right|^{2}\right] .
\end{aligned}
$$

In the same way that we computed the noise correlations in the BCS and BP states, we find the noise correlation in the FFLO state as

$$
\begin{aligned}
G_{\uparrow \downarrow}\left(\mathbf{r}, \mathbf{r}^{\prime}\right)= & A(t)^{4} \sum_{\mathbf{k}} \delta\left(\mathbf{r}-\frac{\hbar t \tilde{\mathbf{k}}}{m}\right) \\
& \times \delta\left(\mathbf{r}^{\prime}+\frac{\hbar t\left(\tilde{\mathbf{k}}^{\prime}-\mathbf{q}\right)}{m}\right) \theta\left(E_{\uparrow, \mathbf{k}, \mathbf{q}}\right) \theta\left(E_{\downarrow, \mathbf{k}, \mathbf{q}}\right) \\
& \times\left|u_{\mathbf{k}, \mathbf{q}}\right|^{2}\left|v_{\mathbf{k}, \mathbf{q}}\right|^{2},
\end{aligned}
$$

$$
\begin{aligned}
G_{\downarrow \uparrow}\left(\mathbf{r}, \mathbf{r}^{\prime}\right)= & A(t)^{4} \sum_{\mathbf{k}} \delta\left(\mathbf{r}+\frac{\hbar t(\tilde{\mathbf{k}}-\mathbf{q})}{m}\right) \\
& \times \delta\left(\mathbf{r}^{\prime}-\frac{\hbar t \widetilde{\mathbf{k}}^{\prime}}{m}\right) \theta\left(E_{\uparrow, \mathbf{k}, \mathbf{q}}\right) \theta\left(E_{\downarrow, \mathbf{k}, \mathbf{q}}\right)\left|u_{\mathbf{k}, \mathbf{q}}\right|^{2}\left|v_{\mathbf{k}, \mathbf{q}}\right|^{2}, \\
G_{\uparrow \uparrow}\left(\mathbf{r}, \mathbf{r}^{\prime}\right)= & -A(t)^{4} \sum_{\mathbf{k}}\left\{\delta\left(\mathbf{r}-\frac{\hbar t \mathbf{k}}{m}\right)\right. \\
& \times \delta\left(\mathbf{r}^{\prime}-\frac{\hbar t \widetilde{\mathbf{k}}^{\prime}}{m}\right) \theta\left(E_{\uparrow, \mathbf{k}, \mathbf{q}}\right) \theta\left(E_{\downarrow, \mathbf{k}, \mathbf{q}}\right)\left|v_{\mathbf{k}, \mathbf{q}}\right|^{4} \\
& \left.+\left[1-\theta\left(E_{\uparrow, \mathbf{k}, \mathbf{q}}\right)\right]\right\}+\delta\left(\mathbf{r}-\mathbf{r}^{\prime}\right)\left\langle\hat{n}_{\uparrow}(\mathbf{r}, t)\right\rangle, \quad(24)
\end{aligned}
$$

Because in the FFLO state momenta $\mathbf{k}$ and $-\mathbf{k}+\mathbf{q}$ are correlated, the points

(a)

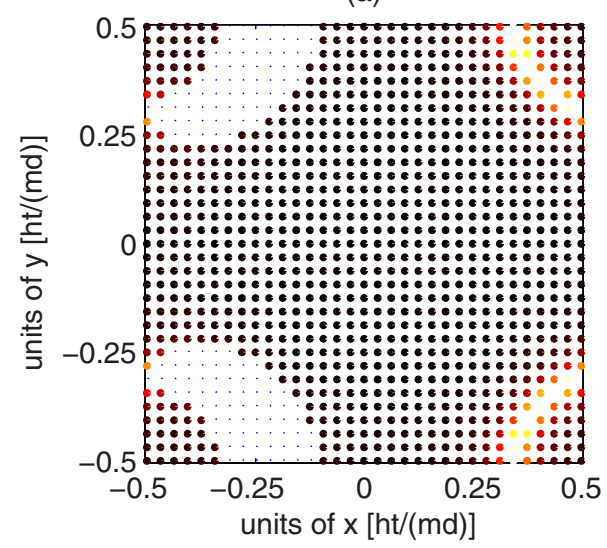

(b)

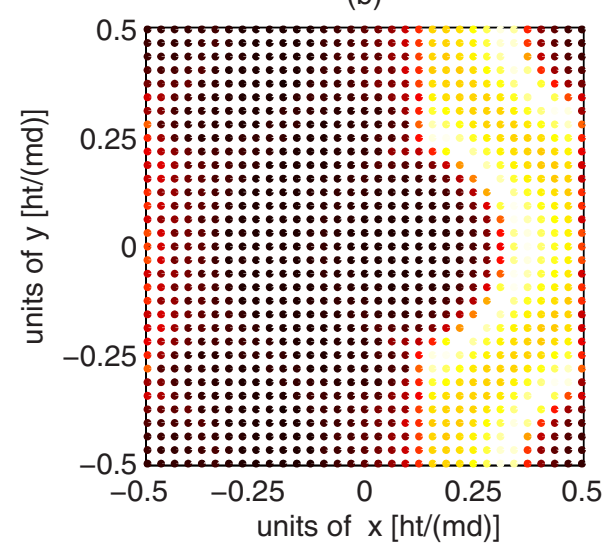

FIG. 4. (Color online) In this figure we show the FFLO-state noise correlation $\left(G_{\uparrow \downarrow}\right)$ at the temperature $T=0$. In (a) we show a cut in the $z=0$ plane while (b) shows the column integrated signal. Parameters we used were $q_{x}=0.25(\pi / d), q_{y}=q_{z}=0$, polarization $P=\left(n_{\uparrow}-n_{\downarrow}\right) /\left(n_{\uparrow}+n_{\downarrow}\right)=0.168, \Delta /(2 J)=0.16$, and $U /(2 J)=-1.86$. In (a) correlations vanish in the dotted areas. We choose $\mathbf{r}+\mathbf{r}^{\prime}$ $-(\hbar t / m) \mathbf{q}=0$ and color coding is again such that light colors imply high peaks and dark colors imply low peaks. 


$$
\mathbf{r}+\mathbf{r}^{\prime}+\frac{\hbar t}{m} \mathbf{q}=\sum_{i=1}^{3} \frac{\hbar 2 n_{i} \pi t \hat{x}_{i}}{m d}
$$

are now correlated in the expanded cloud. Since the FFLO state arises only when there is some polarization in the system, at $T=0$ the FFLO state is always a gapless state, i.e., one of the quasiparticle dispersions $E_{\sigma, \mathbf{k}, \mathbf{q}}$ changes its sign when momentum $\mathbf{k}$ varies.

Multimode FFLO states also leave clear signatures on the noise correlations. For example, for the two mode FFLO state, i.e., the state where the gap is given by $\Delta\left(\mathbf{R}_{i}\right)$ $=\Delta_{0} \cos \left(\mathbf{q} \cdot \mathbf{R}_{i}\right)$, the order parameter can be written as

$$
\begin{aligned}
\Delta\left(\mathbf{R}_{i}\right) & =\Delta_{0} \cos \left(\mathbf{q} \cdot \mathbf{R}_{i}\right)=\Delta_{0}\left(\frac{e^{i \mathbf{q} \cdot \mathbf{R}_{i}}+e^{-i \mathbf{q} \cdot \mathbf{R}_{i}}}{2}\right) \\
& =-U\left\langle\hat{c}_{\uparrow, i} \hat{c}_{\downarrow, i}\right\rangle=\frac{-U}{M} \sum_{\mathbf{k}, \mathbf{k}^{\prime}} e^{-i\left(\mathbf{k}+\mathbf{k}^{\prime}\right) \cdot \mathbf{R}_{i}\left\langle\hat{c}_{\uparrow, \mathbf{k}} \hat{c}_{\downarrow, \mathbf{k}^{\prime}}\right\rangle} \\
& =e^{i \mathbf{q} \cdot \mathbf{R}_{i}} \frac{-U}{M} \sum_{\mathbf{k}}\left\langle\hat{c}_{\uparrow, \mathbf{k}} \hat{c}_{\downarrow,-\mathbf{k}-\mathbf{q}}\right\rangle+e^{-i \mathbf{q} \cdot \mathbf{R}_{i}} \frac{-U}{M} \sum_{\mathbf{k}}\left\langle\hat{c}_{\uparrow, \mathbf{k}} \hat{c}_{\downarrow,-\mathbf{k}+\mathbf{q}}\right\rangle .
\end{aligned}
$$

Therefore the only nonvanishing expectation values in the momentum space are $\left\langle\hat{c}_{\uparrow, \mathbf{k}} \hat{c}_{\downarrow,-\mathbf{k}+\mathbf{q}}\right\rangle$ and $\left\langle\hat{c}_{\uparrow, \mathbf{k}} \hat{c}_{\downarrow,-\mathbf{k}-\mathbf{q}}\right\rangle$. This implies that an $\uparrow$ atom in the momentum state $\mathbf{k}$ is paired with $\downarrow$ atoms in the momentum states $-\mathbf{k}+\mathbf{q}$ and $-\mathbf{k}-\mathbf{q}$. Because in the single mode FFLO state points $\mathbf{r}$ and $-\mathbf{r}+\hbar t \mathbf{q} / m$ are correlated after free expansion, in the two mode FFLO state $\mathbf{r}$ is correlated with $-\mathbf{r}+\hbar t \mathbf{q} / m$ and $-\mathbf{r}-\hbar t \mathbf{q} / m$ after free expansion. For this reason with the two mode FFLO state one can see pronounced correlation peaks when $\mathbf{r}$ $+\mathbf{r}^{\prime} \pm \hbar t \mathbf{q} / m=0$. Actually for the multimode FFLO state, one could use noise correlations to perform a "Fourier analysis" of the periodic order parameter and probe more complicated spatial dependencies than those discussed here.

In Fig. 4 we show the FFLO-state noise correlations $\left(G_{\uparrow \downarrow}\right)$ at zero temperature. Figure 4(a) shows the FFLO-state noise correlation in the $z=0$ plane, and Fig. 4(b) demonstrates the integrated FFLO noise correlation. As one can see, the Fermi surface shift creates asymmetry, which remains also in the integrated signal. Gapless regions of the FFLO state are again reflected as areas without correlation peaks and this is clearly presented in Fig. 4(a).

\section{NOISE CORRELATIONS AT NONZERO TEMPERATURE}

Until now we have assumed zero temperature, but now we generalize our computations to nonzero temperatures. At nonzero temperatures it is useful to write the lowest energy state by using fermionic quasiparticle operators $\hat{\gamma}_{\sigma, \mathbf{k}, \mathbf{q}}$ and $\hat{\gamma}_{\sigma, \mathbf{k}, \mathbf{q}}^{\dagger}$. Creation and annihilation operators can be written in terms of these operators as

$$
\begin{gathered}
\hat{c}_{\uparrow, \mathbf{k}}=u_{\mathbf{k}, \mathbf{q}} \hat{\gamma}_{\uparrow, \mathbf{k}, \mathbf{q}}-v_{\mathbf{k}, \mathbf{q}} \hat{\gamma}_{\downarrow, \mathbf{k}, \mathbf{q}}^{\dagger}, \\
\hat{c}_{\downarrow,-\mathbf{k}+\mathbf{q}}=u_{\mathbf{k}, \mathbf{q}} \hat{\gamma}_{\downarrow, \mathbf{k}, \mathbf{q}}+v_{\mathbf{k}, \mathbf{q}} \hat{\gamma}_{\uparrow, \mathbf{k}, \mathbf{q}}^{\dagger} .
\end{gathered}
$$

Now the lowest energy state ansatz is given by

$$
\left|\Psi_{G S}\right\rangle=\prod_{\mathbf{k}} \hat{\gamma}_{\uparrow, \mathbf{k}, \mathbf{q}}^{\dagger} \hat{\gamma}_{\downarrow, \mathbf{k}, \mathbf{q}}^{\dagger}|0\rangle,
$$

and the lowest energy state is an ideal gas of quasiparticles. In other words, the state in Eq. (26) is the vacuum state for these quasiparticle operators.

The nonvanishing two-operator expectation values in the densities in Eq. (12) at nonzero temperatures are

$$
\begin{gathered}
\left\langle\hat{c}_{\uparrow, \mathbf{k}}^{\dagger} \hat{c}_{\uparrow, \mathbf{k}^{\prime}}\right\rangle=\delta_{\mathbf{k k}^{\prime}}\left\{\left|u_{\mathbf{k}, \mathbf{q}}\right|^{2} f\left(E_{\uparrow, \mathbf{k}, \mathbf{q}}\right)+\left|v_{\mathbf{k}, \mathbf{q}}\right|^{2}\left[1-f\left(E_{\downarrow, \mathbf{k}, \mathbf{q}}\right)\right]\right\}, \\
\left\langle\hat{c}_{\downarrow,-\mathbf{k}+\mathbf{q}}^{\dagger} \hat{c}_{\downarrow,-\mathbf{k}^{\prime}+\mathbf{q}}\right\rangle=\delta_{\mathbf{k k}^{\prime}}\left\{\left|u_{\mathbf{k}, \mathbf{q}}\right|^{2} f\left(E_{\downarrow, \mathbf{k}, \mathbf{q}}\right)+\left|v_{\mathbf{k}, \mathbf{q}}\right|^{2}\left[1-f\left(E_{\uparrow, \mathbf{k}, \mathbf{q}}\right)\right]\right\},
\end{gathered}
$$

where the Fermi-Dirac distribution is given by

$$
f(E)=\frac{1}{e^{E /\left(k_{B} T\right)}+1},
$$

while nonvanishing four-operator expectation values in the noise correlations in Eq. (13) at nonzero temperatures are given by

$$
\begin{aligned}
\left\langle\hat{c}_{\uparrow, \mathbf{k}^{\dagger}}^{\dagger} \hat{c}_{\downarrow,-\mathbf{k}^{\prime}+\mathbf{q}}^{\dagger} \hat{c}_{\downarrow,-\mathbf{k}^{\prime}+\mathbf{q}} \hat{c}_{\uparrow, \mathbf{k}}\right\rangle= & \left|u_{\mathbf{k}, \mathbf{q}}\right|^{2}\left|u_{\mathbf{k}^{\prime}, \mathbf{q}}\right|^{2} f\left(E_{\uparrow, \mathbf{k}, \mathbf{q}}\right) f\left(E_{\downarrow, \mathbf{k}^{\prime}, \mathbf{q}}\right)+\left|u_{\mathbf{k}, \mathbf{q}}\right|^{2}\left|v_{\mathbf{k}^{\prime}, \mathbf{q}}\right|^{2} f\left(E_{\uparrow, \mathbf{k}, \mathbf{q}}\right)\left(1-f\left(E_{\uparrow, \mathbf{k}^{\prime}, \mathbf{q}}\right)\right) \\
& +\left|u_{\mathbf{k}^{\prime}, \mathbf{q}}\right|^{2}\left|v_{\mathbf{k}, \mathbf{q}}\right|^{2} f\left(E_{\downarrow, \mathbf{k}^{\prime}, \mathbf{q}}\right)\left(1-f\left(E_{\downarrow, \mathbf{k}, \mathbf{q}}\right)\right)+\left|v_{\mathbf{k}^{\prime}, \mathbf{q}}\right|^{2}\left|v_{\mathbf{k}, \mathbf{q}}\right|^{2}\left(1-f\left(E_{\uparrow, \mathbf{k}^{\prime}, \mathbf{q}}\right)\right)\left(1-f\left(E_{\downarrow, \mathbf{k}, \mathbf{q}}\right)\right) \\
& +\delta_{\mathbf{k} \mathbf{k}^{\prime}}\left\{\left|u_{\mathbf{k}, \mathbf{q}}\right|^{2}\left|v_{\mathbf{k}, \mathbf{q}}\right|^{2}\left[f\left(E_{\uparrow, \mathbf{k}, \mathbf{q}}\right) f\left(E_{\downarrow, \mathbf{k}, \mathbf{q}}\right)+\left(1-f\left(E_{\uparrow, \mathbf{k}, \mathbf{q}}\right)\right)\left(1-f\left(E_{\downarrow, \mathbf{k}, \mathbf{q}}\right)\right)\right]\right\}\left\langle\hat{c}_{\uparrow, \mathbf{k}}^{\dagger} \hat{c}_{\uparrow, \mathbf{k}^{\prime}}^{\dagger} \hat{c}_{\uparrow, \mathbf{k}^{\prime}} \hat{c}_{\uparrow, \mathbf{k}}\right\rangle \\
= & \left|u_{\mathbf{k}, \mathbf{q}}\right|^{2}\left|u_{\mathbf{k}^{\prime}, \mathbf{q}}\right|^{2} f\left(E_{\uparrow, \mathbf{k}, \mathbf{q}}\right) f\left(E_{\uparrow, \mathbf{k}^{\prime}, \mathbf{q}}\right)+\left|u_{\mathbf{k}, \mathbf{q}}\right|^{2}\left|v_{\mathbf{k}^{\prime}, \mathbf{q}}\right|^{2} f\left(E_{\uparrow, \mathbf{k}, \mathbf{q}}\right)\left(1-f\left(E_{\downarrow, \mathbf{k}^{\prime}, \mathbf{q}}\right)\right) \\
& +\left|u_{\mathbf{k}^{\prime}, \mathbf{q}}\right|^{2}\left|v_{\mathbf{k}, \mathbf{q}}\right|^{2} f\left(E_{\uparrow, \mathbf{k}^{\prime}, \mathbf{q}}\right)\left(1-f\left(E_{\downarrow, \mathbf{k}, \mathbf{q}}\right)\right)+\left|v_{\mathbf{k}^{\prime}, \mathbf{q}}\right|^{2}\left|v_{\mathbf{k}, \mathbf{q}}\right|^{2}\left(1-f\left(E_{\downarrow, \mathbf{k}^{\prime}, \mathbf{q}}\right)\right)\left(1-f\left(E_{\downarrow, \mathbf{k}, \mathbf{q}}\right)\right) \\
& +\delta_{\mathbf{k} \mathbf{k}^{\prime}}\left\{\left|u_{\mathbf{k}, \mathbf{q}}\right|^{2}\left|v_{\mathbf{k}, \mathbf{q}}\right|^{2}\left[f\left(E_{\uparrow, \mathbf{k}, \mathbf{q}}\right) f\left(E_{\downarrow, \mathbf{k}, \mathbf{q}}\right)+\left(1-f\left(E_{\uparrow, \mathbf{k}, \mathbf{q}}\right)\right)\left(1-f\left(E_{\downarrow, \mathbf{k}, \mathbf{q}}\right)\right)\right]\right. \\
& \left.-\left|u_{\mathbf{k}, \mathbf{q}}\right|^{2} f\left(E_{\uparrow, \mathbf{k}, \mathbf{q}}\right)-\left|v_{\mathbf{k}, \mathbf{q}}\right|^{2}\left(1-f\left(E_{\downarrow, \mathbf{k}, \mathbf{q}}\right)\right)\right\} .
\end{aligned}
$$

Because the densities again cancel out most of the terms, the noise correlations turn out to be quite simple, 


$$
\begin{aligned}
G_{\uparrow \downarrow}\left(\mathbf{r}, \mathbf{r}^{\prime}\right)= & A(t)^{4} \sum_{\mathbf{k}}\left[\delta\left(\mathbf{r}-\frac{\hbar t \mathbf{k}}{m}\right) \delta\left(\mathbf{r}^{\prime}+\frac{\hbar t\left(\tilde{\mathbf{k}}^{\prime}-\mathbf{q}\right)}{m}\right)\right. \\
& \times\left\{f\left(E_{\uparrow, \mathbf{k}, \mathbf{q}}\right) f\left(E_{\downarrow, \mathbf{k}, \mathbf{q}}\right)+\left[1-f\left(E_{\uparrow, \mathbf{k}, \mathbf{q}}\right)\right]\right. \\
& \left.\left.\times\left[1-f\left(E_{\downarrow, \mathbf{k}, \mathbf{q}}\right)\right]\right\}\left|u_{\mathbf{k}, \mathbf{q}}\right|^{2}\left|v_{\mathbf{k}, \mathbf{q}}\right|^{2}\right], \\
G_{\downarrow \uparrow}\left(\mathbf{r}, \mathbf{r}^{\prime}\right)= & A(t)^{4} \sum_{\mathbf{k}}\left[\delta\left(\mathbf{r}+\frac{\hbar t(\tilde{\mathbf{k}}-\mathbf{q})}{m}\right) \delta\left(\mathbf{r}^{\prime}-\frac{\hbar t \tilde{\mathbf{k}}^{\prime}}{m}\right)\right. \\
& \times\left\{f\left(E_{\uparrow, \mathbf{k}, \mathbf{q}}\right) f\left(E_{\downarrow, \mathbf{k}, \mathbf{q}}\right)+\left[1-f\left(E_{\uparrow, \mathbf{k}, \mathbf{q}}\right)\right]\right. \\
& \left.\left.\times\left[1-f\left(E_{\downarrow, \mathbf{k}, \mathbf{q}}\right)\right]\right\}\left|u_{\mathbf{k}, \mathbf{q}}\right|^{2}\left|v_{\mathbf{k}, \mathbf{q}}\right|^{2}\right], \\
G_{\uparrow \uparrow}\left(\mathbf{r}, \mathbf{r}^{\prime}\right)= & A(t)^{4} \sum_{\mathbf{k}}\left\{\delta\left(\mathbf{r}-\frac{\hbar t \tilde{\mathbf{k}}}{m}\right) \delta\left(\mathbf{r}^{\prime}-\frac{\hbar t \tilde{\mathbf{k}}^{\prime}}{m}\right)\right. \\
& \times\left\{f\left(E_{\uparrow, \mathbf{k}, \mathbf{q}}\right) f\left(E_{\downarrow, \mathbf{k}, \mathbf{q}}\right)+\left[1-f\left(E_{\uparrow, \mathbf{k}, \mathbf{q}}\right)\right]\right. \\
& \left.\times\left[1-f\left(E_{\downarrow, \mathbf{k}, \mathbf{q}}\right)\right]\right\}\left|u_{\mathbf{k}, \mathbf{q}}\right|^{2}\left|v_{\mathbf{k}, \mathbf{q}}\right|^{2}-\left|u_{\mathbf{k}, \mathbf{q}}\right|^{2} f\left(E_{\uparrow, \mathbf{k}, \mathbf{q}}\right) \\
G_{\downarrow \downarrow}\left(\mathbf{r}, \mathbf{r}^{\prime}\right)= & A(t)^{4} \sum_{\mathbf{k}}\left[\delta\left(\mathbf{r}-\frac{\hbar t(\tilde{\mathbf{k}}-\mathbf{q})}{m}\right) \delta\left(\mathbf{r}^{\prime}-\frac{\hbar t\left(\tilde{\mathbf{k}}^{\prime}-\mathbf{q}\right)}{m}\right)\right. \\
& \times\left\{f\left(E_{\uparrow, \mathbf{k}, \mathbf{q}}\right) f\left(E_{\downarrow, \mathbf{k}, \mathbf{q}}\right)+\left[1-f\left(E_{\uparrow, \mathbf{k}, \mathbf{q}}\right)\right]\right. \\
& \left.\times\left[1-f\left(E_{\downarrow, \mathbf{k}, \mathbf{q}}\right)\right]\right\}\left|u_{\mathbf{k}, \mathbf{q}}\right|^{2}\left|v_{\mathbf{k}, \mathbf{q}}\right|^{2} \\
& \left.\left|v_{\mathbf{k}, \mathbf{q}}\right|^{2}\left[1-f\left(E_{\downarrow, \mathbf{k}, \mathbf{q}}\right)\right]\right\}+\delta\left(\mathbf{r}-\mathbf{r}^{\prime}\right)\left\langle\hat{n}_{\uparrow}(\mathbf{r}, t)\right\rangle, \\
& \\
& \\
&
\end{aligned}
$$

$$
\begin{aligned}
& \left.-\left|u_{\mathbf{k}, \mathbf{q}}\right|^{2} f\left(E_{\downarrow, \mathbf{k}, \mathbf{q}}\right)-\left|v_{\mathbf{k}, \mathbf{q}}\right|^{2}\left[1-f\left(E_{\uparrow, \mathbf{k}, \mathbf{q}}\right)\right]\right] \\
& +\delta\left(\mathbf{r}-\mathbf{r}^{\prime}\right)\left\langle\hat{n}_{\downarrow}(\mathbf{r}, t)\right\rangle .
\end{aligned}
$$

At nonzero temperatures the state is the BCS state if $\mathbf{q}=0$ and the densities are equal, and the BP state if $\mathbf{q}=0$ and the densities are different. Since at finite temperatures the system can support polarization due to thermal effects even if both quasiparticle dispersions are always positive, we call the state the gapless BP state if $\mathbf{q}=0$ and one of the quasiparticle dispersions changes its sign.

Figure 5 demonstrates why the noise correlations are a better indicator of the superfluidity than densities and how the BCS-state noise correlation between the components changes quite substantially when the temperature rises and the gap is reduced. Figure 5(a) shows a cut of the BCS-state density in the $z=0$ plane when the temperature is $k_{B} T /(2 J)$ $=0.196$ (i.e., just below the critical temperature). Figure 5(b) shows a similar result just above the critical temperature. In Figs. 5(c) and 5(d) we compare the column integrated BCSstate noise correlations between the components at the temperatures $k_{B} T /(2 J)=0.0$, and $k_{B} T /(2 J)=0.196$, respectively. By comparing Figs. 5(a) and 5(b), one can see that the densities are almost the same for the BCS state and the normal state. On the other hand, in the normal state the noise correlation is identically zero. Thermal effects are strongly present in the results in Fig. 5(d). While not clear with the parameters chosen here, it is possible that the correlations peak in two areas, close to the center of the figure as well as near the Fermi surface. In the zero temperature result in Fig. 5(c) there are high peaks only near the Fermi surface. The reason for this difference between low and higher temperatures is that when the temperature rises the peak heights around the Fermi surface are reduced with reduced gap while closer to (a)

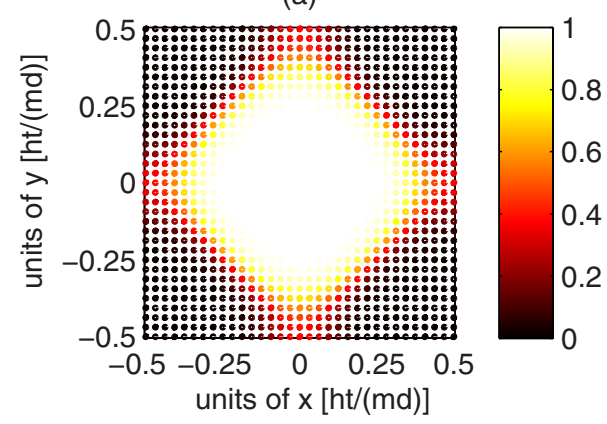

(b)

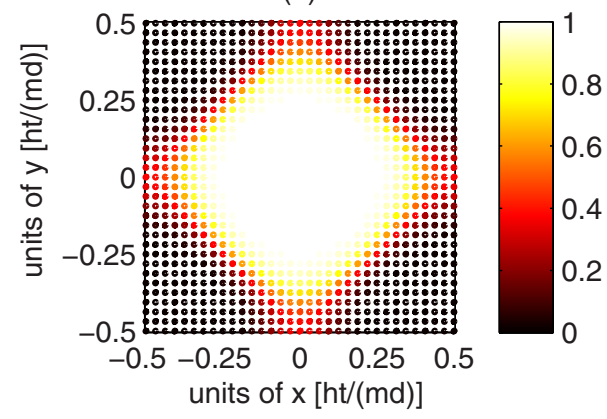

(c)

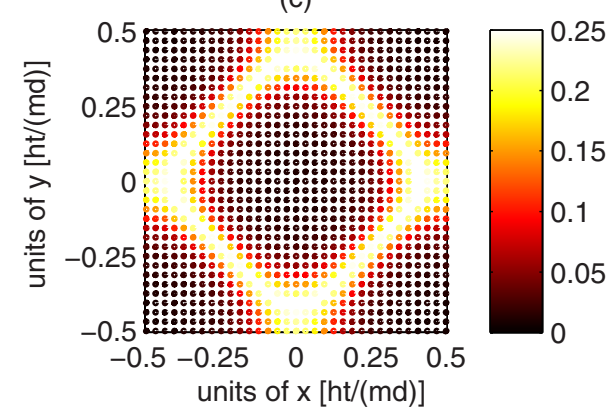

(d)

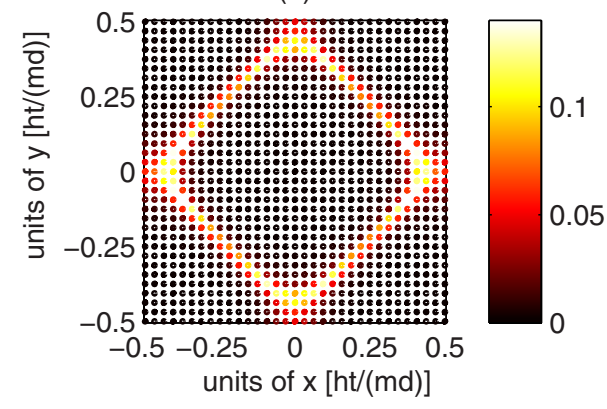

FIG. 5. (Color online) (a) shows a cut of the BCS-state density in the $z=0$ plane when the temperature is $k_{B} T /(2 J)=0.196$ (just below $T_{c}$ ) while figure (b) shows a cut of the normal-state density in the $z=0$ plane when the temperature is $k_{B} T /(2 J)=0.20$ (just above $T_{c}$ ). (c) and (d) show the integrated BCS-state noise correlations between components at temperatures $k_{B} T /(2 J)=0.0$ and $k_{B} T /(2 J)=0.196, \quad$ respectively. The other parameters used were $n_{\uparrow}=n_{\downarrow}=0.20, \quad U /(2 J)=-1.86$, in figures (a) and (d) $\Delta /(2 J)=0.09$, and in figure (c) $\Delta /(2 J)=0.35$. Color coding is again such that light colors imply high peaks and dark colors imply low peaks. 

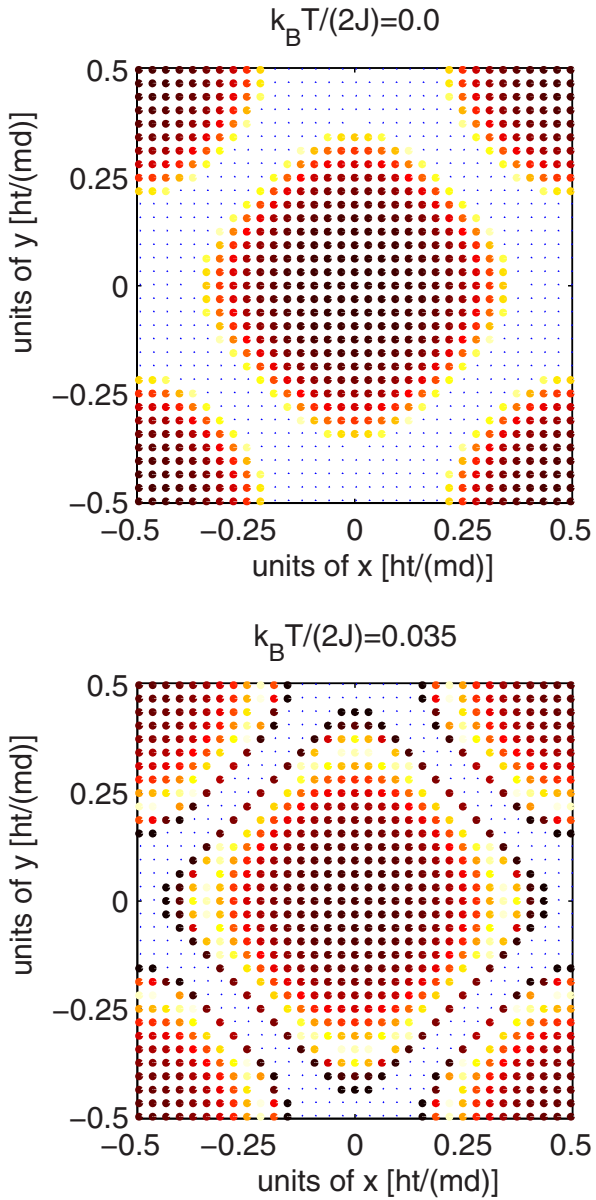
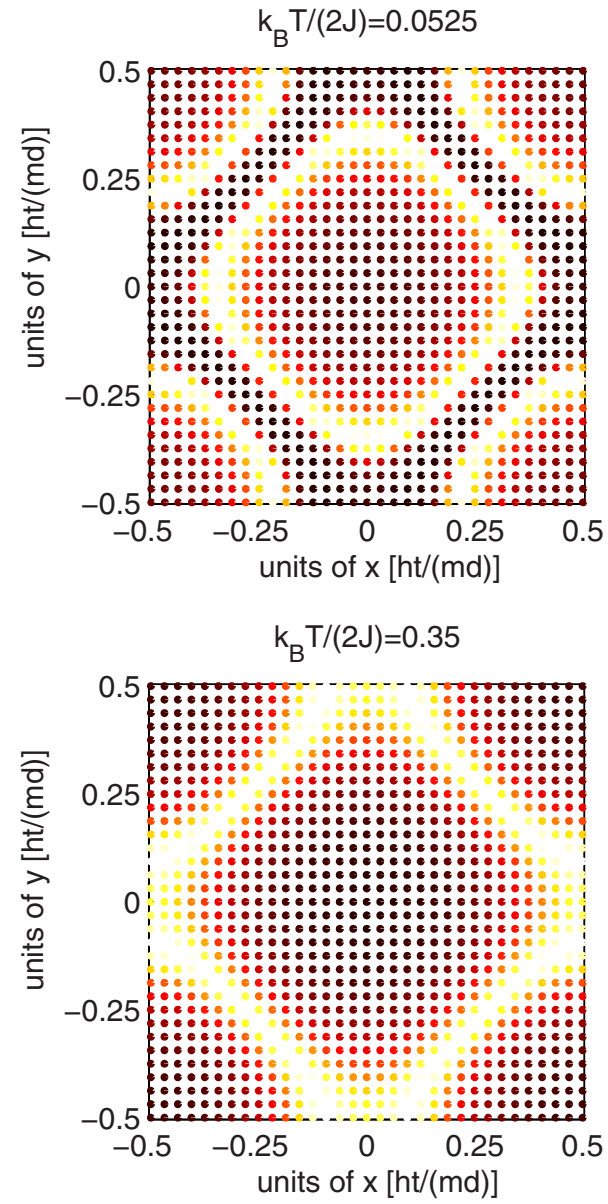

FIG. 6. (Color online) This figure demonstrates how temperature contributes to the noise correlations of the gapless BP state. In these figures the temperature gradually increases, while other things remain equal. The last figure corresponds to the minimum of the grand potential with polarization $P=\left(n_{\uparrow}-n_{\downarrow}\right) /\left(n_{\uparrow}+n_{\downarrow}\right)$ $=0.40$, average filling fraction $\left(n_{\uparrow}+n_{\downarrow}\right) / 2=0.30, \Delta /(2 J)=0.318$, and coupling strength $U /(2 J)=$ -3.0. We have chosen $\mathbf{r}+\mathbf{r}^{\prime}=0$. All figures show the cut in the $z$ $=0$ plane. At the upper row the dotted areas are peakless. Color coding is such that light colors imply high peaks and dark colors imply low peaks. the center, the thermal effects are relatively weaker.

Figure 6 demonstrates how the temperature contributes to the gapless BP-state superfluid noise correlations. As one can see, when temperature rises the peakless areas become smaller and eventually vanish. The reason for this is in the temperature fluctuations, which enables the system to carry polarization even with gapped dispersions. These figures show also that the peakless areas vanish only gradually, when the temperature becomes nonzero.

Finally, Fig. 7 demonstrates how temperature influences the noise correlations of the FFLO state. The states correspond to minima of the free energy at their respective temperatures. Figures 7(a) and 7(b) show an example of what happens to the correlation between components on the $z=0$ plane when temperature rises from $k_{B} T /(2 J)=0.0475$ to $k_{B} T /(2 J)=0.095$. Figures $7(\mathrm{c})$ and $7(\mathrm{~d})$ demonstrate the same for the column integrated noise correlations. As one can see from the figures, sharp areas without correlation peaks again disappear with increasing temperatures. However, the shift in the positions of the peaks persists even at nonzero temperatures.

It might not be easy to detect the gapless states via noise correlations because at the temperature regime where the gapless BP state becomes the lowest energy state, the noise correlations of the gapless BP state and the nongapless BP state appear quite similar. On the other hand, by monitoring the peak heights at $z=0$ (and at $\mathbf{r}^{\prime}=-\mathbf{r}$ ), one can identify the gapless states. The reason for this is that in the gapless BP state the maximum peak height is always smaller than $A(t)^{4} / 8$ while in the nongapless state the maximum is always bigger than this. This can be seen from the fact that the term $\left|u_{\mathbf{k}}\right|^{2}\left|v_{\mathbf{k}}\right|^{2}$ peaks on the Fermi surface corresponding to the average chemical potential. On the other hand, near this surface the peak heights are

$$
\begin{aligned}
&\left|u_{\mathbf{k}}\right|^{2}\left|v_{\mathbf{k}}\right|^{2}\left\{f\left(E_{\uparrow, \mathbf{k}}\right) f\left(E_{\downarrow, \mathbf{k}}\right)+\left[1-f\left(E_{\uparrow, \mathbf{k}}\right)\right]\left[1-f\left(E_{\downarrow, \mathbf{k}}\right)\right]\right\} \\
& \approx \frac{1}{4}\{f(\Delta+\delta \mu / 2) f(\Delta-\delta \mu / 2) \\
&\quad+[1-f(\Delta-\delta \mu / 2)][1-f(\Delta+\delta \mu / 2)]\},
\end{aligned}
$$

where $\delta \mu=\mu_{\uparrow}-\mu_{\downarrow}$ and then the term in the curly brackets is bigger than $1 / 2$, when $\Delta \pm \delta \mu / 2>0$ (and the state is gapped) and otherwise always lower than $1 / 2$.

In Fig. 8 we show the height of the maximum of peak height as a function of the polarization [Fig. 8(a)] and as a function of chemical potential difference [Fig. 8(b)]. Figure 8(a) shows that when the polarization $P$ rises then the maximum peak height becomes smaller and drops suddenly to zero when the gas becomes normal. With our parameters this happens when the polarization is about 0.35 . By inspecting Fig. 8(b) it is clear that when the BP state is gapless, i.e., when $\delta \mu>2 \Delta$, the maximum peak height is indeed smaller than $1 / 8$, a result which was made plausible above. 
(a)

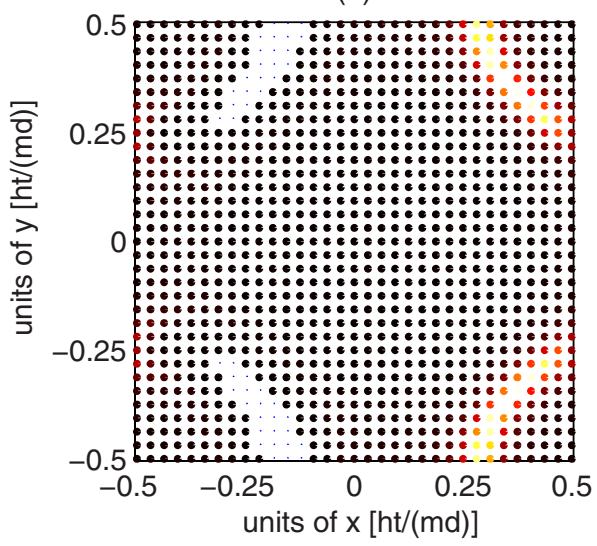

(b)

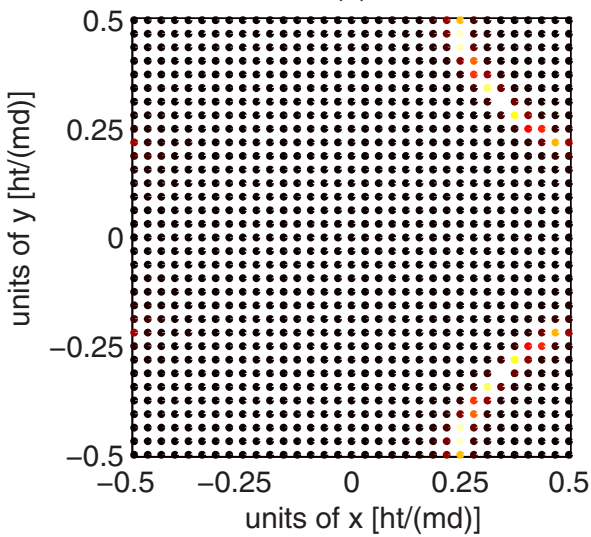

(c)

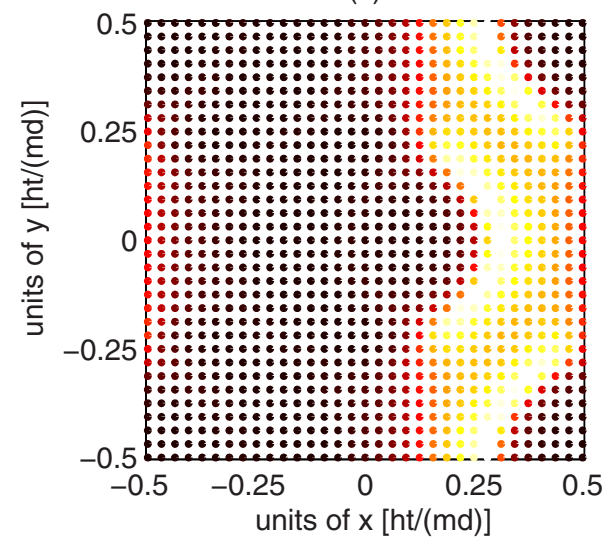

(d)

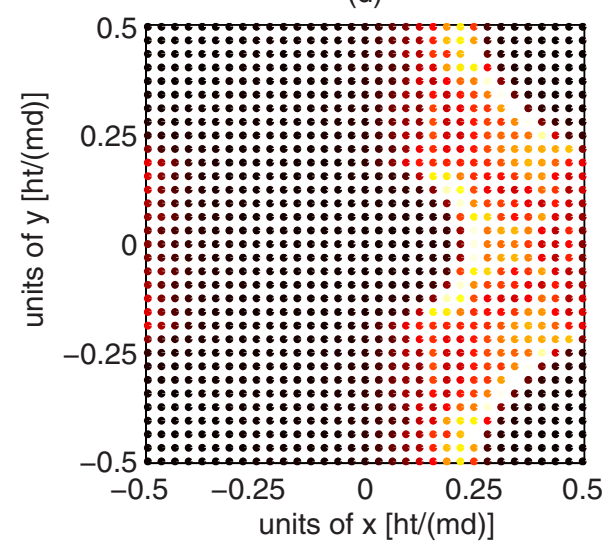

FIG. 7. (Color online) Finite temperature noise correlations $\left(G_{\uparrow \downarrow}\right)$ of the FFLO state. (a) and (b) show cuts in the $z=0$ plane, while (c) and (d) are the column integrated correlations. (a) and (c) were calculated at $k_{B} T /(2 J)$ $=0.0475$ and $(\mathrm{b})$ and $(\mathrm{d})$ were calculated at $k_{B} T /(2 J)=0.095$. In all figures we used the coupling strength $U /(2 J)=-1.86$. In (a) and (c) $P=0.208, \quad\left(n_{\uparrow}+n_{\downarrow}\right) / 2=0.46$, $q_{x}=0.26(\pi / d), \quad q_{y}=q_{z}=0$, and $\Delta /(2 J)=0.14$. In (b) and (d) $P$ $=0.24, \quad\left(n_{\uparrow}+n_{\downarrow}\right) / 2=0.37, \quad q_{x}$ $=0.21(\pi / d), \quad q_{y}=q_{z}=0, \quad$ and $\Delta /(2 J)=0.046$. We have chosen $\mathbf{r}+\mathbf{r}^{\prime}-(\hbar t / m) \mathbf{q}=0$. In (a) correlations vanish in the dotted areas. Color coding is again such that light colors imply high peaks and dark colors imply low peaks.

\section{NOISE CORRELATIONS IN A ONE-DIMENSIONAL LATTICE}

We call the lattice one-dimensional (1D) when $J_{x} \gg J_{y}$ $=J_{z}$. In other words a 1D lattice can be realized with a threedimensional (3D) lattice with a very strong confinement in two directions and weaker confinement in the third one. Since the FFLO-type states are more favorable in 1D than they are in 3D [31-34] and the differences between different phases can be more pronounced in $1 \mathrm{D}$ than in $3 \mathrm{D}$, we now consider the noise correlations in 1D lattices. We assume that the gas in a 1D lattice is released in only one direction (in our case $x$ direction). The released gas creates a set of almost identical one-dimensional tubes.

Figure 9 shows an example of the phase diagram in a 1D lattice [34]. It is clear that the FFLO region is remarkably large and occupies the entire superfluid region at $T=0$. On the other hand, the expected region for phase separation between the normal gas and the BCS state (red) is dramatically smaller than in 3D [22]. When the temperature rises there are phase transitions between the FFLO state and, depending on polarization, phase separation, the BCS or BP states, or the normal gas phases. In Fig. 9 we also denote by dashed lines those values of temperature and polarization we have used in our following noise correlation computations.

Figure 10 demonstrates how temperature contributes to the noise correlations in a $1 \mathrm{D}$ lattice. As is clear, when the temperature becomes nonzero the gapless region does not vanish suddenly. Also, there is a phase transition between the
FFLO state and the gapless BP state when the temperature is (with our parameters) between $k_{B} T /\left(2 J_{x}\right)=0.095$ and $k_{B} T /\left(2 J_{x}\right)=0.104$. One can identify this phase transition from the fact that in the FFLO state the noise correlation is not symmetric with respect to $x=0$, while the gapless BP-state noise correlation is symmetric. For the two-mode FFLO state we expect a mirror image of the FFLO-state noise correlation shown here to appear. This mirror image would correspond to correlations with the pair momenta $-q_{x}$. The figure which has been calculated at highest temperature $\left[k_{B} T /\left(2 J_{x}\right)\right.$ $=0.104]$ shows that the gapless BP-state noise correlation is again always below $1 / 8$.

In Fig. 11 we show how the polarization contributes to the noise correlations in a $1 \mathrm{D}$ lattice. When the polarization is between $P=0.32$ and $P=0.35$ the nongapless state becomes gapless and as one can see that the maximum of the gapped $\mathrm{BP}$-state noise correlation is always bigger than $1 / 8$. When the polarization is between $P=0.35$ and $P=0.48$, there is a phase transition from the BP-superfluid state to the FFLO state which is visible in the pronounced asymmetry.

In one-dimensional systems, exact solutions for the problem of interacting fermions exist [43-45]. Moreover, density matrix renormalization group (DMRG) calculations can be applied in one dimension to provide exact numerical results. It is known that mean-field results deviate considerably from the exact ones in one dimension. In Ref. [46], mean-field calculations within the one-dimensional Hubbard model (which is the case we consider) were compared to the exact solutions [43-45]. The ground state energies were found to 


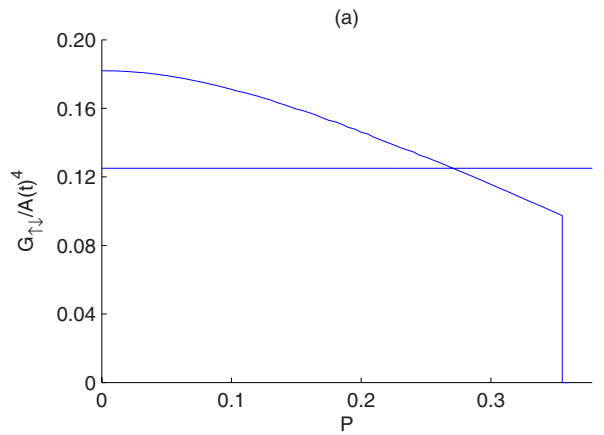

(b)

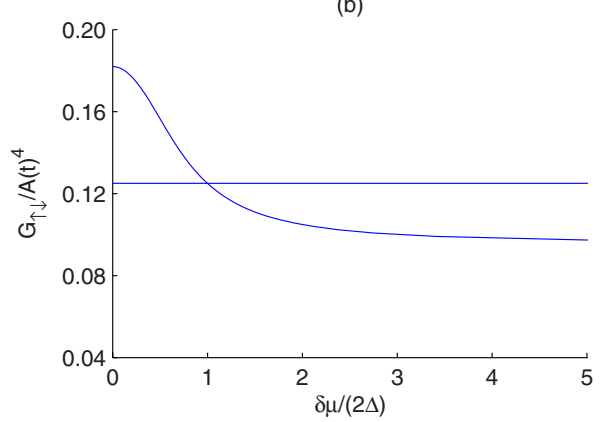

FIG. 8. (Color online) This figure demonstrates how the polarization contributes to the maximum peak height. (a) shows the maximum peak height as a function of the polarization, while (b) shows the maximum peak height as a function of the chemical potential difference $\left(\mu_{\uparrow}-\mu_{\uparrow}\right) /(2 \Delta)=\delta \mu /(2 \Delta)$. We used the parameters $k_{B} T /(2 J)=0.45$ and an average filling fraction $\left(n_{\uparrow}+n_{\downarrow}\right) / 2$ $=0.30$. We have canceled the time dependence of the maximum peak height by dividing the maximum peak height by the scaling factor $A(t)^{4}$. The horizontal lines show the value $1 / 8$.

be very similar, deviating at maximum a few percent. However, the mean-field calculations were found to overestimate the excitation gap energy considerably, typically by a factor of 1.5-3. The deviations are at their strongest at half filling. These results imply that while the low temperature structure in the phase diagram of Fig. 9 is not likely to be affected by the mean-field approximation, the critical temperatures are probably higher than given by the exact solution. The dominance of the FFLO state at low temperatures and for a large range of polarizations, as shown by the mean-field results in Fig. 9, is firmly established also by recent exact $[47,48]$ and DMRG numerical studies [35,49]. Note that the reference [35] considers also noise correlations. It is interesting that our results for the FFLO correlations at zero temperature are quite similar with the more accurate computations using DMRG by Lüscher et al. [35]. In particular, both results show an area in momentum space where correlation peaks are absent as well as pronounced asymmetry. In the single mode FFLO ansatz the signals from both $\pm q$ are naturally missing while DMRG computation is done for a trapped geometry and does show strong correlations corresponding to both $\pm q$. In summary, our results on the structure of the noise correlations for different states in 1D are essentially not affected by the use of mean-field approximation; only the precise values of temperatures where these states occur are to be determined more carefully.

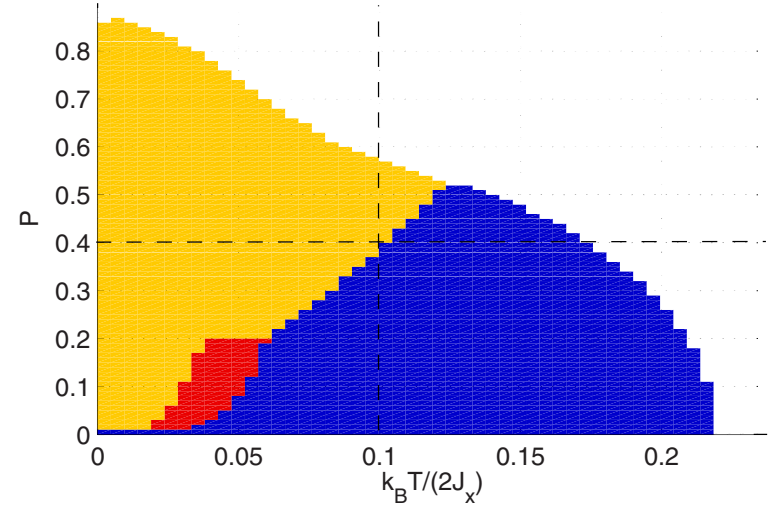

FIG. 9. (Color online) The phase diagram of Fermi gas in an effectively 1D lattice [34]. Colors (or shading): BCS or PB states =blue or dark gray, FFLO=yellow or light gray, phase separation $=$ red or gray, and normal gas $=$ white. The parameters are such that the average filling fraction $\left(n_{\uparrow}+n_{\downarrow}\right) / 2=0.2, J_{x}=0.07 E_{r}$, and $U=$ $-0.2 E_{r}$. The dashed lines show the values $P=0.40$ and $k_{B} T /\left(2 J_{x}\right)$ $=0.104$ used in Figs. 10 and 11 .

\section{CONCLUSIONS}

In this paper we have presented, at the mean-field level, the noise correlations of the two component Fermi gas in an optical lattice. We have shown that the noise correlations are a promising way to detect different phases in optical lattices. The different superfluid phases (BCS, FFLO, and BP) can be distinguished via the noise correlations and by mapping the correlations more extensively; a "Fourier analysis" of the multimode FFLO state is possible and could be used to reveal the structure of the more complicated periodic order parameters. We computed the noise correlations also at nonzero temperatures and demonstrated that the differences between the correlations of different states can persist at finite temperatures and some qualitative features are insensitive to temperature. Also, regions of gapless quasiparticle dispersions can be visible in the noise correlation signals.

Other probes also exist. By letting the gas expand freely, one can measure the momentum distribution by simply imaging the density distribution of the expanded cloud. From this momentum distribution one can, in principle, infer some important properties of the system. For example, one could detect the gapless BP state in this way [50]. However, this method does not appear to be a promising way to detect modulated phases [35]. Spectroscopic means can also be considered. For example, using two-photon Bragg spectroscopy $[51,52]$ one can probe the systems response at specific momenta and in that way gain information on the quasiparticle excitations [53-55]. We are not aware of anyone analyzing this problem for fermions in optical lattices. rf spectroscopy has been proposed [56] for detecting a spatially nonuniform FFLO order parameter: an additional spectral peak appears due to quasiparticles at the nodes of the order parameter. One very appealing property of noise correlation signals is their sensitivity to interactions and pairing effects. If pairing does not take place, noise correlations between different components vanish. This is in contrast to other methods where the influence of the other component is less 

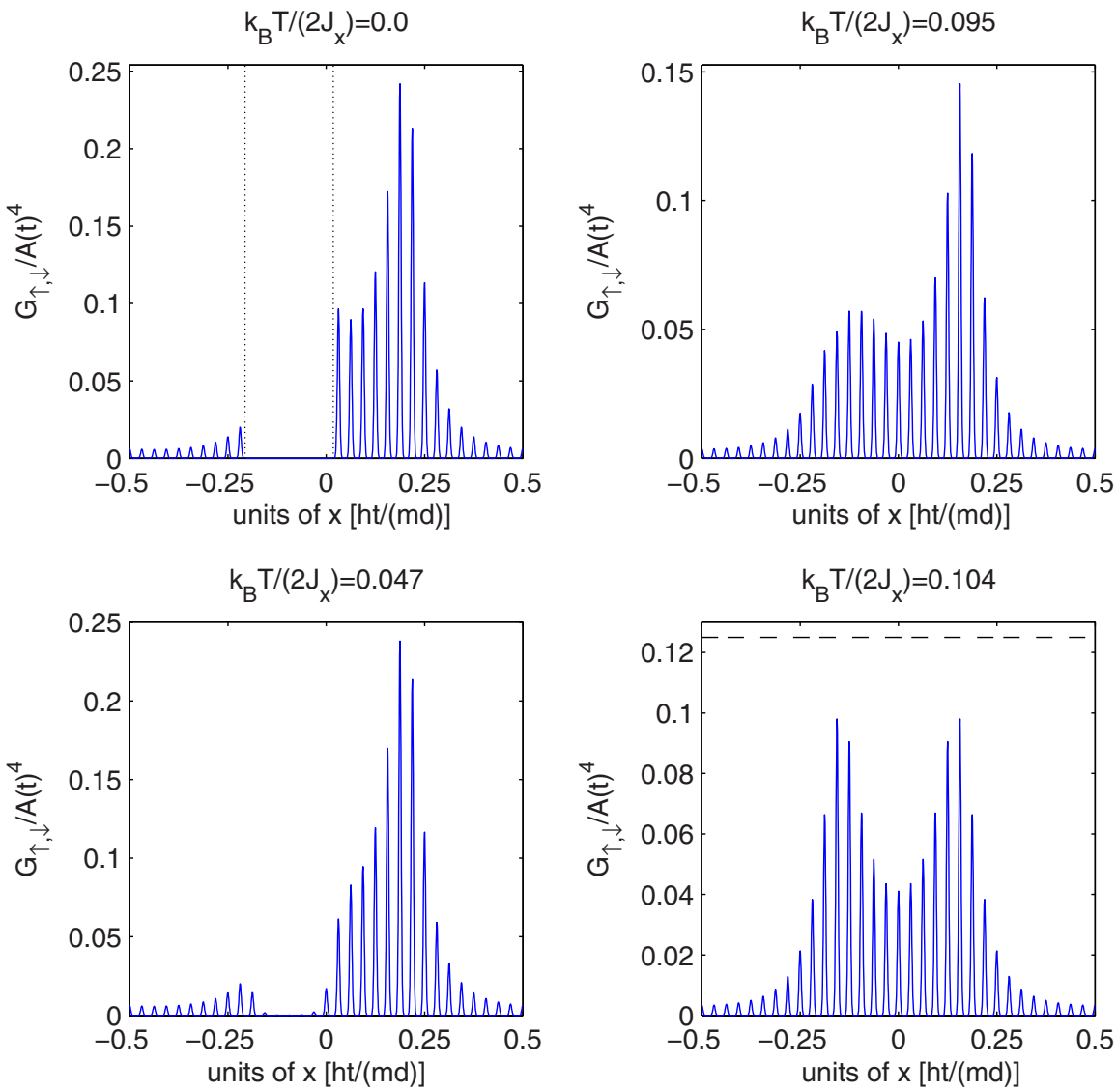

FIG. 10. (Color online) This figure demonstrates how temperature contributes to the noise correlations in a 1D lattice. In these figures we gradually increase the temperature while keeping a polarization $P=0.40$ and an average filling fraction $\left(n_{\uparrow}+n_{\downarrow}\right) / 2=0.20$ fixed. The figures in the upper row and bottom left show the FFLOstate noise correlations. The figure in the bottom right shows the gapless BP-state noise correlation. In the first figure the dashed lines show the gapless region and in the bottom right figure the dashed line shows the value $1 / 8$. We choose $x+x^{\prime}+\hbar t q_{x} / m=0$ in all these figures. In the BPstate $q_{x}=0$.
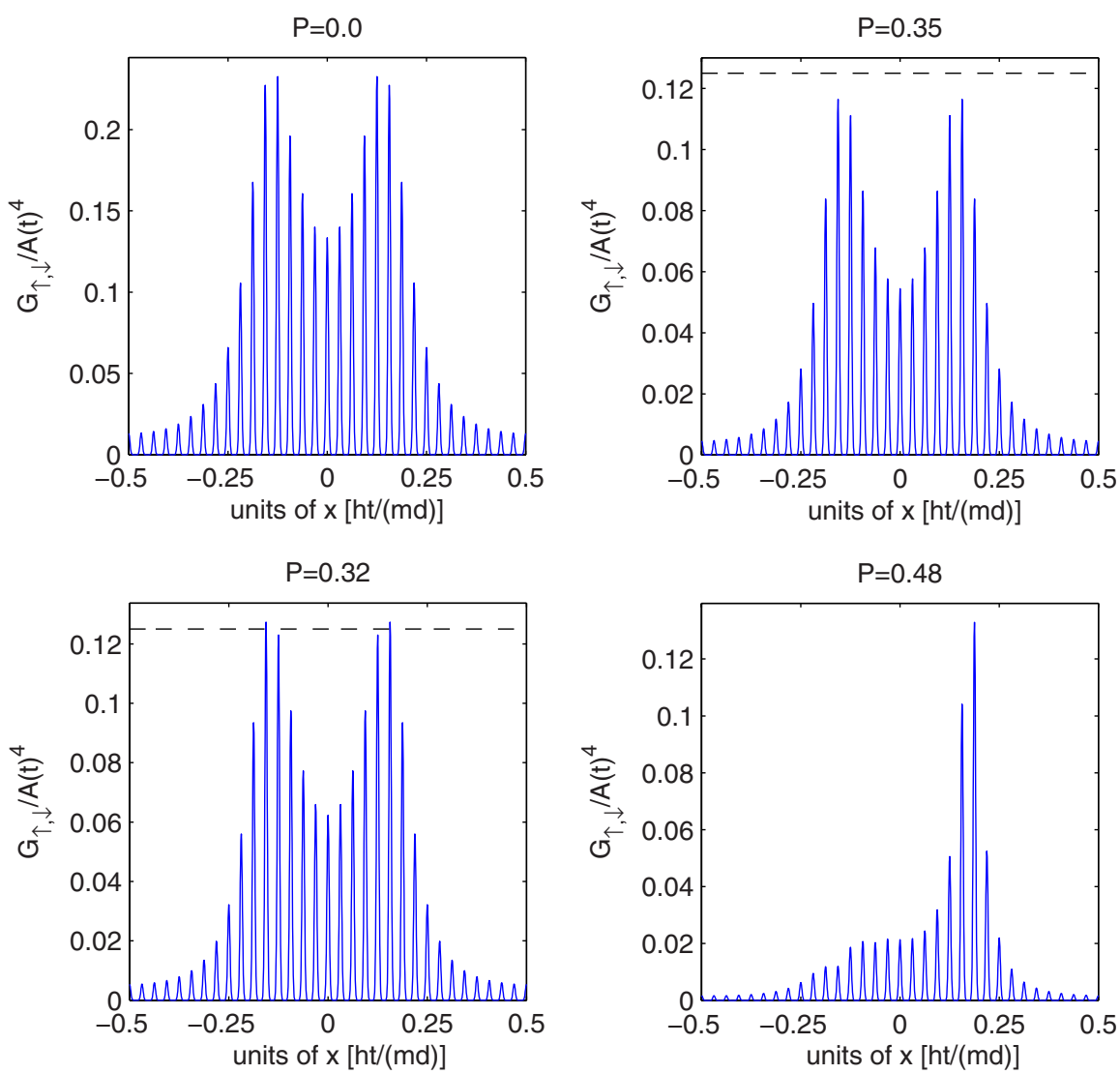

FIG. 11. (Color online) This figure shows how polarization contributes to the noise correlations in a 1D lattice. Here we gradually increase the polarization while keeping the temperature fixed at $k_{B} T /\left(2 J_{x}\right)=0.104$ and an average filling fraction at $\left(n_{\uparrow}\right.$ $\left.+n_{1}\right) / 2=0.20$. The upper left figure shows the BCS-state noise correlation and the upper right figure shows the gapped BP-state noise correlations. The bottom left figure shows the gapless BP-state noise correlation and the figure on the bottom right is the FFLO-state noise correlation. The dashed line shows the value $1 / 8$. We choose $x+x^{\prime}+\hbar t q_{x} / m=0$ in all these figures. In the BCS and the BP states $q_{x}=0$. 
direct. Noise correlations can be especially useful in distinguishing between possible pseudogap effects and superfluidity. Pseudogap implies noncondensed pairs, then noise correlations between momenta $\mathbf{k}+\mathbf{q}$ and $-\mathbf{k}+\mathbf{q}$ appear not only for a single or a few discrete values of $\mathbf{q}$, but for a thermally distributed set of $\mathbf{q}$ values. The appearance of correlations only for a certain $\mathbf{q}$ signals condensation of the pairs.

Density-density correlations of a one-dimensional system were also discussed quite extensively and were shown to contain very clear information on the structure of the pairwave function as well as on the quasiparticle dispersions. In one-dimensional systems, order parameter modulations are favored and possible problems with signals becoming smoothed out by column integration can be avoided. For this reason they are very attractive candidates to observe FFLOtype states experimentally. However, how to balance the requirements of sufficiently reduced dimensionality with sufficient phase coherence [33] is to a large extent still unclear.

It is physically possible that in a three-dimensional system a phase separation occurs between the normal state and the BCS state if the system is polarized $[41,42,57]$. This means that the normal state exists in one part of the lattice and the
BCS state exists in another part of the lattice, typically in the core if a harmonic trap potential is included. However, other more exotic possibilities also exist [34]. Interestingly, the phase separation between the normal gas and a paired state could be visible in the noise correlations between components. This follows from the fact that the noise correlations between components in the normal state vanish, whereas in the paired state the correlations are at their strongest around the Fermi momentum. In a lattice superimposed by a trap the local density, and therefore the local Fermi momentum, is different in different areas of the gas. This may allow one to identify spatial phase separation and shell structures of normal and paired states from the freely expanded cloud where momentum has been mapped into position.

\section{ACKNOWLEDGMENTS}

This work was supported by the National Graduate School in Materials Physics, Academy of Finland and ESF/ EURYI (Projects No. 115020, No. 213362, No. 121157, and No. 207083). See www.esf.org/euryi.
[1] M. W. Zwierlein, A. Schirotzek, C. H. Schunck, and W. Ketterle, Science 311, 492 (2006).

[2] G. B. Partridge, W. Li, R. I. Kamar, Y. Liao, and R. G. Hulet, Science 311, 503 (2006).

[3] M. W. Zwierlein, C. H. Schunck, A. Schirotzek, and W. Ketterle, Nature (London) 442, 54 (2006).

[4] Y. Shin, M. W. Zwierlein, C. H. Schunck, A. Schirotzek, and W. Ketterle, Phys. Rev. Lett. 97, 030401 (2006).

[5] G. B. Partridge, W. Li, Y. A. Liao, R. G. Hulet, M. Haque, and H. T. C. Stoof, Phys. Rev. Lett. 97, 190407 (2006).

[6] Y. Shin, C. H. Schunck, A. Schirotzek, and W. Ketterle, Nature (London) 451, 689 (2008).

[7] P. Fulde and R. A. Ferrell, Phys. Rev. 135, A550 (1964).

[8] A. I. Larkin and Y. N. Ovchinnikov, Zh. Eksp. Teor. Fiz. 47, 1136 (1964).

[9] G. Sarma, J. Phys. Chem. Solids 24, 1029 (1963).

[10] W. V. Liu and F. Wilczek, Phys. Rev. Lett. 90, 047002 (2003).

[11] R. Casalbuoni and G. Nardulli, Rev. Mod. Phys. 76, 263 (2004).

[12] C. Orzel, A. K. Tuchman, M. L. Fenselau, M. Yasuda, and M. A. Kasevich, Science 291, 2386 (2001).

[13] M. Greiner, I. Bloch, O. Mandel, T. W. Hänsch, and T. Esslinger, Phys. Rev. Lett. 87, 160405 (2001).

[14] S. Burger, F. S. Cataliotti, C. Fort, F. Minardi, M. Inguscio, M. L. Chiofalo, and M. P. Tosi, Phys. Rev. Lett. 86, 4447 (2001).

[15] Z. Hadzibabic, S. Stock, B. Battelier, V. Bretin, and J. Dalibard, Phys. Rev. Lett. 93, 180403 (2004).

[16] W. Hofstetter, J. I. Cirac, P. Zoller, E. Demler, and M. D. Lukin, Phys. Rev. Lett. 89, 220407 (2002).

[17] G. Orso and G. V. Shlyapnikov, Phys. Rev. Lett. 95, 260402 (2005).

[18] L. P. Pitaevskii, S. Stringari, and G. Orso, Phys. Rev. A 71, 053602 (2005).
[19] M. Iskin and C. A. R. Sa de Melo, Phys. Rev. Lett. 99, 080403 (2007).

[20] T. Koponen, J.-P. Martikainen, J. Kinnunen, and P. Törmä, Phys. Rev. A 73, 033620 (2006).

[21] T. Koponen, J. Kinnunen, J.-P. Martikainen, L. M. Jensen, and P. Törmä, New J. Phys. 8, 179 (2006).

[22] T. K. Koponen, T. Paananen, J.-P. Martikainen, and P. Törmä, Phys. Rev. Lett. 99, 120403 (2007).

[23] J. K. Chin, D. E. Miller, Y. Liu, C. Stan, W. Setiawan, C. Sanner, K. Xu, and W. Ketterle, Nature (London) 443, 961 (2006).

[24] M. Köhl, H. Moritz, T. Stöferle, K. Günter, and T. Esslinger, Phys. Rev. Lett. 94, 080403 (2005).

[25] T. Stöferle, H. Moritz, K. Günter, M. Köhl, and T. Esslinger, Phys. Rev. Lett. 96, 030401 (2006).

[26] K. Günter, T. Stöferle, H. Moritz, M. Köhl, and T. Esslinger, Phys. Rev. Lett. 95, 230401 (2005).

[27] E. Altman, E. Demler, and M. D. Lukin, Phys. Rev. A 70, 013603 (2004).

[28] S. Fölling, F. Gerbier, A. Widera, O. Mandel, T. Gericke, and I. Bloch, Nature (London) 434, 481 (2005).

[29] T. Rom, T. Best, D. van Oosten, U. Schneider, S. Foelling, B. Paredes, and I. Bloch, Nature (London) 444, 733 (2006).

[30] M. Greiner, C. A. Regal, J. T. Stewart, and D. S. Jin, Phys. Rev. Lett. 94, 110401 (2005).

[31] K. Machida and H. Nakanishi, Phys. Rev. B 30, 122 (1984).

[32] X.-J. Liu, H. Hu, and P. D. Drummond, Phys. Rev. A 76, 043605 (2007).

[33] M. M. Parish, S. K. Baur, E. J. Mueller, and D. A. Huse, Phys. Rev. Lett. 99, 250403 (2007).

[34] T. K. Koponen, T. Paananen, J.-P. Martikainen, M. R. Bakhtiari, and P. Törmä, e-print arXiv:0711.4065v2.

[35] A. Lüscher, R. M. Noack, and A. Laeuchli, e-print arXiv:0712.1808v2. 
[36] I. Bloch, J. Dalibard, and W. Zwerger, e-print arXiv:0704.3011.

[37] D. Jaksch, C. Bruder, J. I. Cirac, C. W. Gardiner, and P. Zoller, Phys. Rev. Lett. 81, 3108 (1998).

[38] J. Bardeen, L. N. Cooper, and J. R. Schrieffer, Phys. Rev. 108, 1175 (1957).

[39] D. E. Sheehy and L. Radzihovsky, Ann. Phys. 322, 1790 (2007).

[40] B. M. Andersen and G. M. Bruun, Phys. Rev. A 76, 041602(R) (2007).

[41] P. F. Bedaque, H. Caldas, and G. Rupak, Phys. Rev. Lett. 91, 247002 (2003).

[42] J.-P. Martikainen, Phys. Rev. A 74, 013602 (2006).

[43] M. Gaudin, Phys. Lett. 24A, 55 (1967).

[44] C. N. Yang, Phys. Rev. Lett. 19, 1312 (1967).

[45] E. H. Lieb and F. Y. Wu, Phys. Rev. Lett. 20, 1445 (1968).

[46] F. Marsiglio, Phys. Rev. B 55, 575 (1997).

[47] G. Orso, Phys. Rev. Lett. 98, 070402 (2007).

[48] H. Hu, X.-J. Liu, and P. D. Drummond, Phys. Rev. Lett. 98,
070403 (2007).

[49] A. E. Feiguin and F. Heidrich-Meisner, Phys. Rev. B 76, 220508(R) (2007).

[50] W. Yi and L.-M. Duan, Phys. Rev. Lett. 97, 120401 (2006).

[51] J. Stenger, S. Inouye, A. P. Chikkatur, D. M. Stamper-Kurn, D. E. Pritchard, and W. Ketterle, Phys. Rev. Lett. 82, 4569 (1999).

[52] J. M. Vogels, K. Xu, C. Raman, J. R. Abo-Shaeer, and W. Ketterle, Phys. Rev. Lett. 88, 060402 (2002).

[53] D. van Oosten, D. B. M. Dickerscheid, B. Farid, P. van der Straten, and H. T. C. Stoof, Phys. Rev. A 71, 021601(R) (2005).

[54] K. J. Challis, R. J. Ballagh, and C. W. Gardiner, Phys. Rev. Lett. 98, 093002 (2007).

[55] G. M. Bruun and G. Baym, Phys. Rev. A 74, 033623 (2006).

[56] J. Kinnunen, L. M. Jensen, and P. Törmä, Phys. Rev. Lett. 96, 110403 (2006).

[57] M. Haque and H. T. C. Stoof, Phys. Rev. A 74, 011602(R) (2006). 